\title{
Emerging roles of ATG proteins and membrane lipids in autophagosome formation
}

\author{
Taki Nishimura (1) and Sharon A. Tooze (D)
}

\begin{abstract}
Autophagosome biogenesis is a dynamic membrane event, which is executed by the sequential function of autophagy-related (ATG) proteins. Upon autophagy induction, a cup-shaped membrane structure appears in the cytoplasm, then elongates sequestering cytoplasmic materials, and finally forms a closed double membrane autophagosome. However, how this complex vesicle formation event is strictly controlled and achieved is still enigmatic. Recently, there is accumulating evidence showing that some ATG proteins have the ability to directly interact with membranes, transfer lipids between membranes and regulate lipid metabolism. A novel role for various membrane lipids in autophagosome formation is also emerging. Here, we highlight past and recent key findings on the function of ATG proteins related to autophagosome biogenesis and consider how ATG proteins control this dynamic membrane formation event to organize the autophagosome by collaborating with membrane lipids.
\end{abstract}

\section{Introduction}

Under nutrient limited conditions cells survive by degrading their cellular contents to maintain homeostasis, energy levels, and building blocks. Macro-autophagy (hereafter, autophagy) is an intracellular degradation system that delivers cytoplasmic materials to the lysosome/vacuole. In response to autophagy induction, a cupshaped membrane structure termed the phagophore (also known as the isolation membrane) appears in the cytoplasm. Once the phagophore elongates enough to accommodate its substrates, it closes and seals to form a double membrane structure, called an autophagosome (Fig. 1a). Initially autophagy was mainly analyzed in rat liver hepatocytes by electron microscopy ${ }^{1-3}$ and biochemical enzyme assays ${ }^{4,5}$. However, after the discovery of autophagy in the yeast Saccharomyces cerevisiae ${ }^{6}$, and the isolation of the yeast $A T G$ (autophagy-related) genes $^{7-9}$, research into autophagy has been transformed from a descriptive phenomenon into a biochemical and molecular field using model organisms, cell culture systems

Correspondence: Taki Nishimura (taki.nishimura@crick.ac.uk) or

Sharon A. Tooze (sharon.tooze@crick.ac.uk)

${ }^{1}$ Molecular Cell Biology of Autophagy, The Francis Crick Institute, London NW1 1AT, UK and in vitro reconstitution approaches. Core Atg/ATG proteins are functionally categorized into discrete units: the Atg1/ULK complex, the class III phosphatidylinositol 3-kinase (PI3K) complex, the Atg2-Atg18/WIPI4 complex, Atg9 vesicle, the Atg12 conjugation system, including ATG12-5-16L1 and WIPI2B, and the Atg8/LC3 conjugation system ${ }^{10-12}$. This molecular understanding of the autophagic machinery has led to the discovery of its importance in tumorigenesis ${ }^{13}$, mammalian development $^{14,15}$, lipid metabolism ${ }^{16}$, degradation of intracellular pathogens ${ }^{17}$, and neurodegeneration ${ }^{18,19}$. In recent years, diverse physiological and pathological roles of autophagy have been uncovered ${ }^{20}$.

Autophagosome formation is driven by the ATG proteins, but during this dynamic membrane remodeling lipids are major constituents of autophagic membranes. Although the lipid composition of autophagosomes remains obscure, phosphatidylinositol 3-phosphate (PI3P) and phosphatidylethanolamine (PE) are crucial for autophagosome formation. The role of PI3P in autophagy was guided by a study showing the inhibitory activity of 3-methyladenine in rat hepatocytes ${ }^{21}$. Subsequently, it was shown that class III PI3K is a target of 3methyladenine ${ }^{22,23}$. In line with this, Vps34 was 


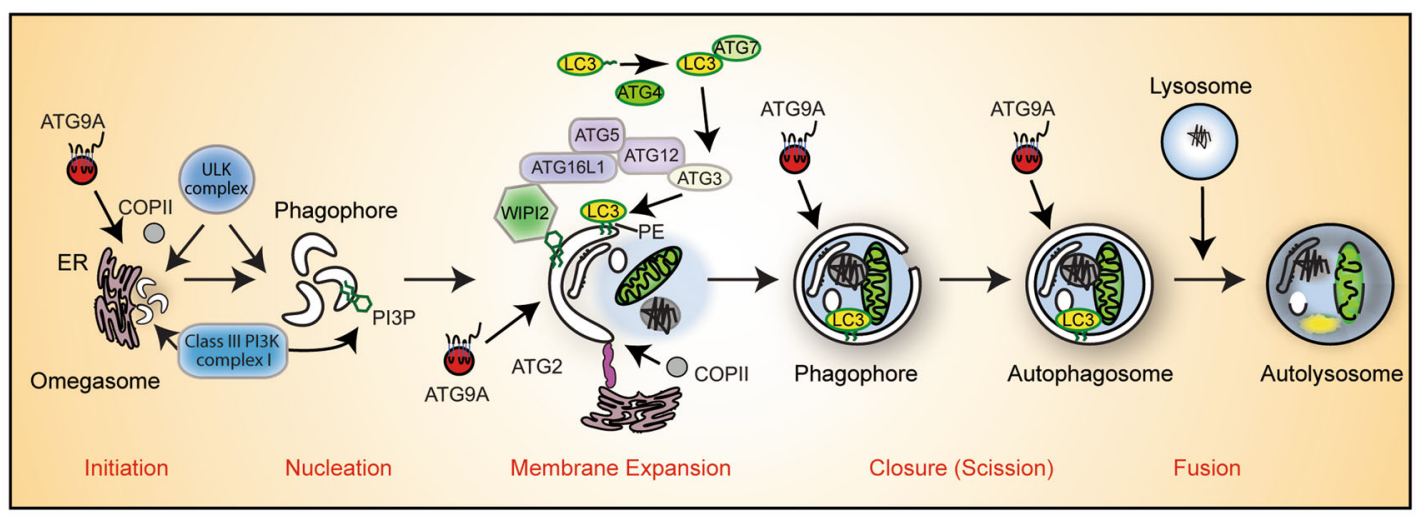

b

\begin{tabular}{|c|c|c|c|c|c|c|c|c|}
\hline & \multirow{2}{*}{$\begin{array}{l}\text { Initiation } \\
\text { complex }\end{array}$} & \multirow{2}{*}{$\begin{array}{c}\mathrm{ER} \\
\text { (puncta) }\end{array}$} & \multirow{2}{*}{$\begin{array}{c}\text { ERES } \\
\text { (puncta) }\end{array}$} & \multirow{2}{*}{$\begin{array}{l}\text { ERGIC } \\
\text { (puncta) }\end{array}$} & \multirow{2}{*}{ Omegasome } & \multicolumn{2}{|c|}{ Phagophore } & \multirow{2}{*}{ Autophagosome } \\
\hline & & & & & & edge & body & \\
\hline ULK1 & + & + & + & + & + & $+?$ & ++ & $-/+$ \\
\hline ATG13 & + & + & $-1+$ & + & + & $+?$ & ++ & $-/+$ \\
\hline FIP200 & + & + & + & + & + & $+?$ & ++ & $-1+$ \\
\hline ATG9A & $-1+$ & $-/+$ & $-1+$ & $-1+$ & $-1+$ & $-1+$ & $-/+$ & $-/+$ \\
\hline ATG14L & - & + & $-/+?$ & + & + & $+?$ & ++ & $-/+$ \\
\hline ATG2 & - & + & $-/+?$ & $+?$ & $+?$ & ++ & $-/+$ & $-/+$ \\
\hline WIPI2B & - & $-?$ & $-?$ & $+?$ & + & +++ & +++ & $-/+$ \\
\hline ATG12-5-16L1 & - & $-?$ & $-?$ & $+?$ & + & +++ & +++ & $-/+$ \\
\hline LC3-PE & - & - & $-?$ & $+?$ & + & +++ & +++ & +++ \\
\hline
\end{tabular}

C

\begin{tabular}{|c|c|c|c|c|c|c|}
\hline & \multicolumn{5}{|c|}{ Preautophagosomal structure (PAS) } & \multirow{3}{*}{ Autophagosome } \\
\hline & \multirow{2}{*}{$\begin{array}{c}\text { Initiation complex } \\
\text { (a punctate structure) }\end{array}$} & \multirow{2}{*}{$\begin{array}{c}\text { ER/ERES } \\
\text { (a punctate structure) }\end{array}$} & \multirow{2}{*}{$\begin{array}{l}\text { Vacuole-phagophore } \\
\text { contact sites }\end{array}$} & \multicolumn{2}{|c|}{ Phagophore } & \\
\hline & & & & edge & body & \\
\hline Atg1 & + & $-1+$ & ++ & + & ++ & ++ \\
\hline Atg13 & + & $-1+$ & +++ & $-1+$ & + & $-1+$ \\
\hline Atg17 & + & $-1+$ & +++ & + & $-1+$ & $-1+$ \\
\hline Atg9 & $-/+$ & $-1+$ & + & ++ & $-1+$ & + \\
\hline Atg14 & - & $-1+$ & +++ & $-1+$ & + & $-/+$ \\
\hline Atg18 & - & $-1+$ & + & ++ & $-1+$ & $-1+$ \\
\hline Atg2 & - & + & + & ++ & $-1+$ & $-/+$ \\
\hline Atg12-5-16 & - & $-?$ & ++ & $-1+$ & ++ & $-1+$ \\
\hline Atg3 & - & $-?$ & + & $-1+$ & ++ & $-1+$ \\
\hline Atg8-PE & - & $-/+$ & ++ & +++ & +++ & +++ \\
\hline
\end{tabular}

Fig. 1 ATG/Atg proteins control dynamic membrane events during autophagosome biogenesis. a Autophagosome formation can be dissected into five different steps: initiation, nucleation, membrane expansion, closure, and fusion. $\mathbf{b}, \mathbf{c}$ The intracellular distribution of ATG/Atg proteins under starvation-induced autophagy in mammalian (b) $)^{32,34-36,48,56,58,97,127,161,172}$ and yeast cells $(\mathbf{c})^{33,46,47,55,126,130,154,155}$. Their localizations are categorized into five groups: -, not detectable; $-/+$, transient; + , weakly detectable; ++ , easily detectable; +++ , clearly detectable. Note that ATG/ Atg proteins show punctate structures on the ER-related membranes rather than a typical ER-like pattern. ERES ER exit sites, ERGIC ER-Golgi intermediate compartment. 
identified as a PI3K in yeast ${ }^{24}$ and mammals ${ }^{25}$, and a binding partner of Vps30/Atg6 $6^{26}$. Finally, the Vps34Vps15-Vps30/Atg6-Atg14 complex was characterized as an autophagy-specific class III PI3K complex ${ }^{26}$. PI3P is a minor lipid, but its formation is crucial for membrane recruitment of ATG proteins and the early stage of autophagosome formation (Fig. 1a). In contrast, PE is a major phospholipid in eukaryotic cells. Under starved conditions, Atg8/LC3/GABARAP proteins are bound to autophagic membranes through covalent conjugation to PE (Fig. 1a), which is a hallmark of autophagy ${ }^{27}$. Although Atg8/LC3/GABARAP proteins can be also conjugated to phosphatidylserine (PS) in vitro, $\mathrm{PE}$ is the major target of Atg8/LC3/GABARAP conjugation in vivo ${ }^{28,29}$.

Considering ATG protein recruitment to autophagic membranes is both spatially and temporally controlled, changes in the amount of PI3P and PE on autophagic membranes are unlikely to fully explain how ATG proteins execute their functions and accomplish autophagosome biogenesis. Here, we review key studies and recent findings on the molecular machinery underlying autophagosome formation, and focus on the recent literature investigating the co-operation of ATG proteins with membrane lipids during autophagy, and the emerging roles of membrane lipids in autophagosome formation.

\section{The ULK/Atg1 complex: an initiator of autophagosome formation}

The ULK/Atg1 complex is one of the earliest factors to target to membrane structures at the phagophore formation site (Fig. 1b, c). This complex composed of four components (ULK1/2, ATG13, FIP200/RB1CC1, and ATG101) in mammals (Fig. 2a), and five components (Atg1, Atg13, Atg17, Atg29, and Atg31) in yeast (Fig. 2b), is a pivotal signal transducer of autophagy. So far, the identification of ULK/Atg1 substrates has been largely confined to ones involved in autophagy $y^{30,31}$. There is accumulating evidence showing that membrane recruitment of ULK/Atg1 complex at the initiation stage does not require downstream ATG proteins. In genetic hierarchical analyses, the puncta formation of ULK/Atg1 complex is independent of other Atg complexes in both yeast and mammalian cells ${ }^{32,33}$. A comprehensive biochemical analysis has shown that ULK complex components are bound to membranes even in the absence of downstream ATG proteins $^{34}$. Importantly, under starved conditions the formation of the ULK complex puncta can occur during inhibition of PI3P synthesis although the size and lifespan of puncta are reduced ${ }^{34-36}$. Thus, the ULK/Atg1 complex is first recruited to membrane structures independently of PI3P and downstream ATG proteins, and then its membrane localization is stabilized by $\mathrm{PI}^{3} \mathrm{P}^{36}$. Yet, which components of the ULK/Atg1 complex act as determinants for membrane recruitment is unclear.
To date, there have been several reports on the direct membrane association of the ULK/Atg1 complex components. A putative lipid-binding domain has been identified in ULK/Atg1 proteins. The C-terminus of ULK1 associates with cell membranes (Fig. 2a, red line) ${ }^{37}$, and in line with this, the Atg1 EAT domain (Fig. 2b, red line), a region that corresponds to the ULK1 C-terminus, binds to liposomes with a preference for small highly curved vesicles $^{38,39}$, suggesting that the ULK/Atg1 C-terminus serves as a membrane curvature sensor. However, the Atg1 EAT domain needs to interact with Atg13 to induce autophagy ${ }^{40,41}$, which hinders the membrane association of Atg1 EAT domain ${ }^{38}$. Therefore, it is unclear if the Atg1 EAT domain in fully assembled Atg1 complex can associate with membranes. A recent paper has proposed that the Atg13-free Atg1 EAT domain is involved in later stages of autophagosome biogenesis ${ }^{42}$.

ATG13/Atg13 also has a putative lipid-binding region. Membrane binding of human ATG13 is regulated by a cluster of arginine/lysine residues at the $\mathrm{N}$-terminus (Fig. 2a, blue line) via an electrostatic interaction with negatively charged phospholipids, such as phosphatidic acid and phosphatidylinositol phosphates (PIPs) ${ }^{36}$. Similarly, the C-terminus of yeast Atg13 has positively charged residues (Fig. 2b, blue line) and directly binds to liposomes containing negatively charged lipids in vitro. Thus, the lipid binding ability of Atg13 seems to be conserved from yeast to human, although the domain carrying the cluster of arginine/lysine residues is not conserved ${ }^{39,43}$.

Secretory pathway proteins can participate in membrane targeting of the ULK/Atg1 complex components. In yeast, a Rab GTPase Ypt1, which is essential for ER-Golgi and Golgi traffic, recruits Atg1 to the preautophagosomal structures (PAS) via an interaction with the $\mathrm{N}$-terminus of Atg1 (Fig. 2b), although other Atg1 complex components, Atg13 and Atg17, are localized to the PAS independently of Ypt $1^{44}$. The initiation step has also been spatially linked to COPII vesicles, which mediate ER-to-Golgi transport $^{45-48}$. In mammalian cells, SEC12, the activator of COPII assembly, is associated with the FIP200 C-terminus, although this interaction is mainly required for FIP200 function in the remodeling of ER exit sites (ERES), specialized ER regions for COPII vesicle formation ${ }^{48}$. A recent study has revealed that ULK1 and FIP200 interact with integral ER proteins, VAP proteins (VAPA and VAPB), which can tether the ER to other cell membranes at membrane contact sites ${ }^{49}$. VAP proteins directly bind FFAT (two phenylalanines in an acidic tract) motifcontaining proteins to the $\mathrm{ER}^{50}$. Consistent with this, ULK1 and FIP200 have functional FFAT motifs, suggesting that membrane association of ULK1 and FIP200 is regulated by VAP proteins. Therefore, Ypt1, SEC12, and VAPs facilitate the initiation of autophagy by recruitment of the ULK complex components. However, given that 


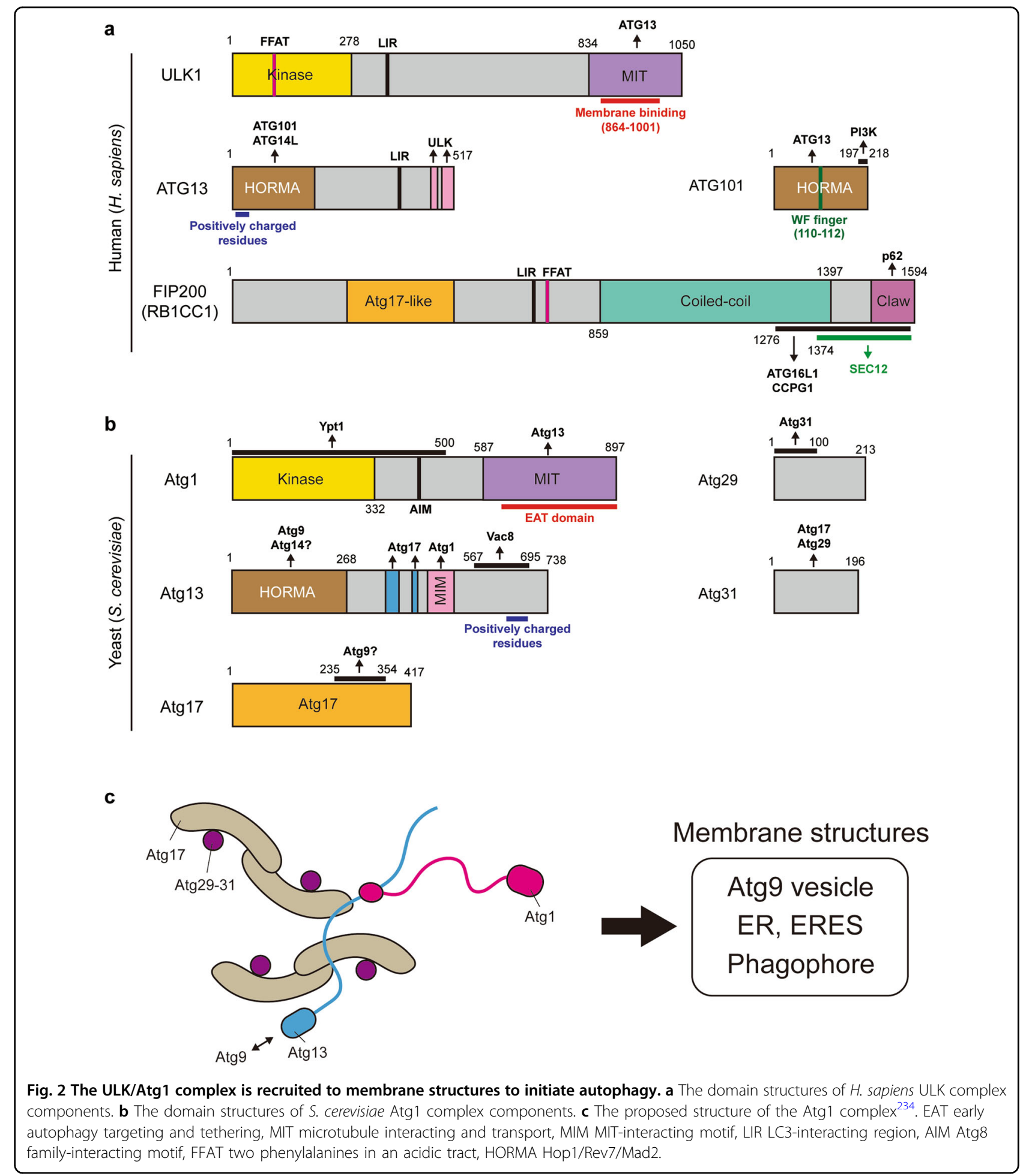

autophagic phenotypes caused by the inhibition of these interactions are partial ${ }^{44,48,49}$, we suggest the leading players for the recruitment of the Atg1/ULK complex are still missing (Fig. 2c).

Late stage recruitment of ULK/Atg1 complex during autophagosome formation requires LC3/GABARAP/Atg8 proteins. ULK/Atg1 complex components have a LC3/ GABARAP/ATG8-interacting (LIR/AIM) motif and bind to ATG8 family proteins (Fig. 2a, b $)^{51-53}$. These interactions have been proposed to be involved in autophagosome maturation and/or a negative feedback by degrading the ULK/Atg1 complex via autophagy. 


\section{Atg9 vesicles: a membrane source for autophagosome formation}

ATG9A/Atg9 is a multi-spanning membrane protein essential for the initiation of autophagosome formation (Fig. 1a). ATG9A/Atg9 cycles between different organelle compartments via vesicular transport pathways and delivered to the autophagosome formation site in response to induction of autophagy. In yeast Atg9 localizes to vesicular and tubular structures at the PAS under starved condition ${ }^{54}$. Atg9-containing vesicles, the diameter of which are $30-60 \mathrm{~nm}$, are highly mobile within the cytoplasm ${ }^{55}$. As a small part of yeast Atg9 localizes to the autophagosomal outer membrane, it has been proposed that Atg9 vesicles become a seed membrane for phagophore formation in yeast ${ }^{55}$. In contrast, mammalian ATG9A is not obviously incorporated into autophagosomal membranes. Rather, ATG9A is found on clusters of vesicles and/or tubules in the vicinity of phagophores and transiently associated with the autophagosomal membranes $^{32,56-58}$. Accordingly, it is thought that ATG9A supplies key components, such as proteins and lipids, to the autophagosomal membranes by transient association. Despite the apparent differences in the localization of ATG9A during autophagy initiation, the two models proposed in yeast and mammalian cells are not mutually exclusive.

Recently, the machinery sorting ATG9A/Atg9 to different locations in the cell has become increasingly clear. Atg9 distribution is partly regulated by the Atg1 complex via its physical interactions with other Atg proteins (Fig. 3a). Cytoplasmic regions of Atg9 interact with Atg17 ${ }^{39,59}$. The $\mathrm{N}$-terminus of Atg9 also binds to Atg13 HORMA domain, which facilitates recruitment of Atg9 vesicles to the PAS ${ }^{60}$. Atg9 is phosphorylated by Atg1 kinase, and this phosphorylation is required for recruitment of downstream factors such as Atg18 and Atg2, while it does not affect the PAS recruitment of $A \operatorname{tg} 9^{61}$. As in yeast, the trafficking of ATG9A is dependent on the ULK complex ${ }^{37,62,63}$ although further analysis is needed to confirm whether ATG9A directly interacts with the ULK complex.

ATG9A/Atg9 traffic is also controlled by the vesicular transport machinery. The N-terminal tail of ATG9A contains sorting signals recognized by adaptor protein (AP) complexes and interacts with AP-1, AP-2 and AP-4 complex subunits (Fig. 3b, c) ${ }^{63-65}$. ATG9A distribution is altered in AP-2 knockdown ${ }^{66}$ and AP-4 KO cells ${ }^{65,67-69}$ : AP-2 likely mediates transport of ATG9A from the plasma membrane and recycling endosomes, while AP-4 regulates exit from the TGN. Yeast Atg9 does not have adaptor complex recognition signals, but instead Atg23 and Atg27 function to export Atg9 into Golgi-derived vesicles $^{70}$. Rab GTPases, GEFs (guanine nucleotide exchange factor), and GAPs (GTPase-activating protein) also control ATG9A/Atg9 trafficking, mainly between the
ERGIC, Golgi, and recycling endosomes. Ypt1 is recruited to Atg9 vesicles in yeast ${ }^{71,72}$. Similarly, ATG9A is distributed into RAB1- and RAB11-positive compartments $^{73,74}$. Trs85/TRAPPC8, a subunit of TRAPPIII complex (a GEF for Ypt1/RAB1) and Trs130, a subunit of TRAPPII complex (a GEF for Ypt31/Ypt32) regulate ATG9A/Atg9 traffic presumably by mediating Rab-GTP activity $^{73,75,76}$. RabGAPs, TBC domain-containing proteins (TBC1D5 and TBC1D14), also contribute to ATG9A cycling ${ }^{66,73}$. TBC1D14, which has no detectable GAP activity, has been proposed to function as a effector for GTP-loaded RAB11 ${ }^{57}$. TBC1D14 controls RAB1 activity by coupling with RAB11-GTP and the TRAPPIII complex $^{73}$. Finally, N-BAR-containing proteins BIF-1 and sorting nexin SNX18 are involved in tubulation and fission of ATG9A-positive recycling endosomes by recruiting dynamin $2^{77,78}$. p38IP, an ATG9A interactor, is required for the redistribution of ATG9A in response to starvation $^{79}$. Interestingly, phosphatidylinositol 4-phosphate (PI4P) may also regulate Atg9 traffic as a phosphatidylinositol 4-phosphate kinase Pik1 is essential for Atg9 exit from the $\mathrm{Golgi}^{80}$.

The essential role of ATG9A/Atg9 in autophagy remains elusive, but a recent study has revealed a close relationship between ATG9A vesicle and PI4P metabolism $^{81}$. The BAR-domain-containing protein Arfaptin-2 and two PI4Ks (PI4KII $\alpha$ and PI4KIII $\beta$ ) have been identified as components of ATG9A vesicles. ATG9A vesicles transport PI4KIII $\beta$ to the ER membrane promoting PI4P production at the initiation site, which facilitates recruitment of the ULK complex and initiation of autophagy. In this model, Arfaptin-2 has been proposed to serve as a regulator of ATG9A vesicles by modulating ATG9A exit from the Golgi complex ${ }^{81}$. PI4KII $\alpha$ may also be delivered by ATG9A vesicles to provide PI4P for later stages of autophagosome maturation ${ }^{82}$. Thus, a key role of ATG9A in autophagy may be to supply PI4P to autophagosomal membranes. Very recently, using single-particle cryoelectron microscopy the structure of Arabidopsis thaliana ATG9 was reported at sub-nanometer resolution ${ }^{83}$. Future structural studies at even higher resolution are needed to reveal some of the unresolved roles of ATG9A/ Atg9.

\section{The class III PI3K complex I (PI3KC3-C1): a PI3P generator at the initiation site}

The class III PI3K complex I (PI3KC3-C1), that is essential for the nucleation of autophagosomes, consists of Vps34/VPS34, Vps15/p150, Vps30/BECN1, and Atg14/ ATG14L (Fig. 4a, b) ${ }^{26,84-86}$. A fifth subunit Atg38/NRBF2 facilitates the PI3KC3-C1 complex formation and further induces PI3KC3-C1 dimerization ${ }^{87-92}$. To generate PI3P, the PI3KC3-C1 needs to directly interact with membranes and recognize the substrate lipid, phosphatidylinositol 


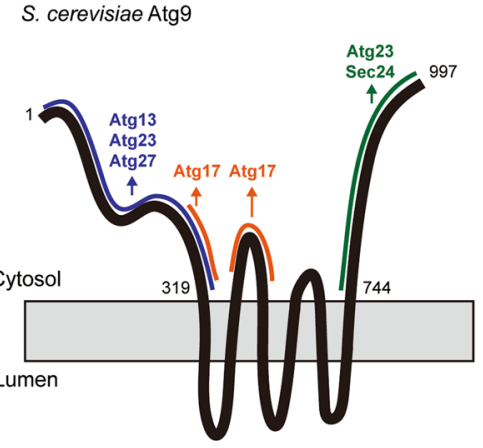

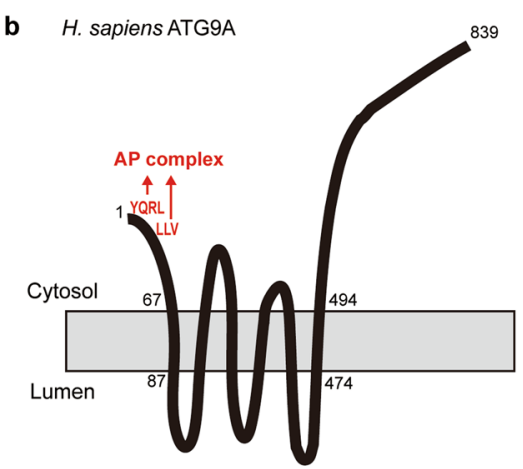

c

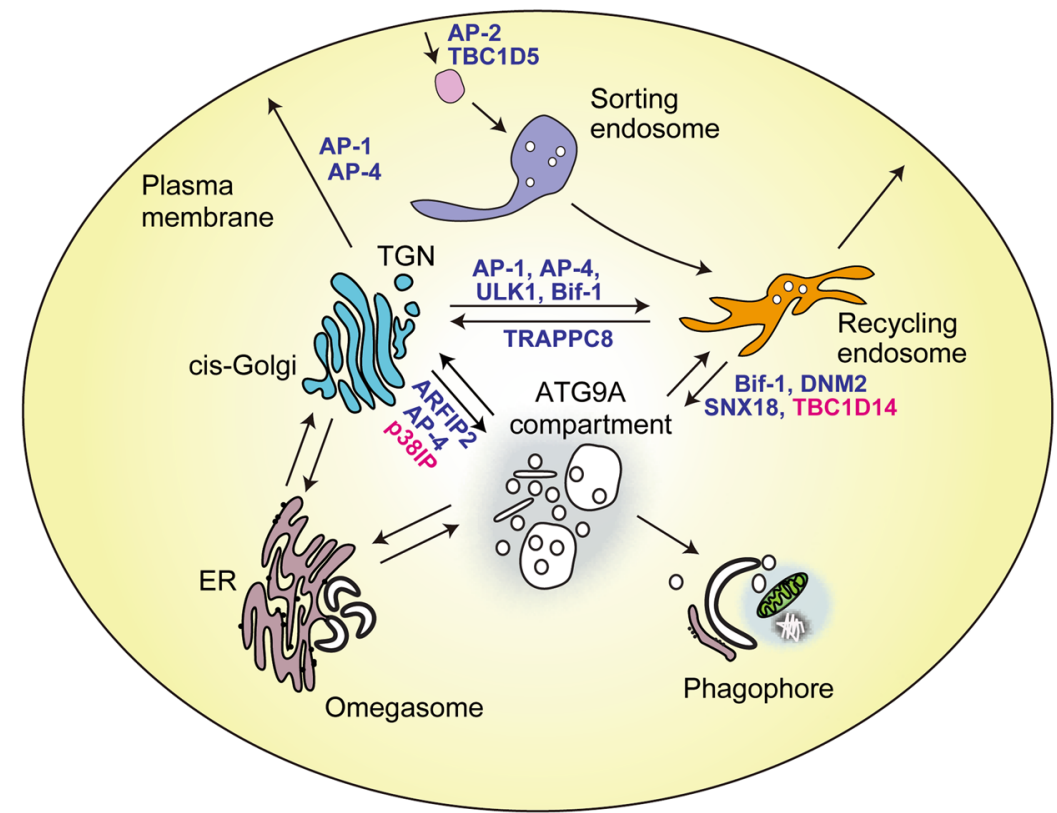

Fig. 3 Atg9/ATG9A vesicles work as a membrane source for autophagosome formation. $\mathbf{a}, \mathbf{b}$ The domain structure of S. cerevisiae Atg9 (a) and H. sapiens ATG9A (b) proteins. c Mammalian ATG9A cycles between different organelle compartments via vesicular transport pathways. Positive and negative regulators are shown in blue and red, respectively. AP adaptor protein, ARFIP2 arfaptin-2, SNX18 sorting nexin 18, DNM2 dynamin 2, TBC1D14 TBC1 domain family member 14, TRAPPC8 trafficking protein particle complex 8, p38IP p38-interacting protein.

(PI). Several studies have revealed that among the PI3KC3-C1 complex components Vps34/VPS34, Vps30/ BECN1, and ATG14L have lipid-binding domains (Fig. 4a, b, red lines $)^{93-96}$. ATG14L plays a key role in an ERtargeting of the PI3KC3-C1 ${ }^{97}$.

Recent studies by single-particle electron microscopy and crystal structural analysis have provided many insights into how lipid-binding domains individually contribute to the membrane association of the PI3KC3C1 complex ${ }^{95,98,99}$. The PI3KC3-C1 complex demonstrates a two-armed V-shaped architecture (Fig. 4c). One arm contains the helical and lipid kinase domains of Vps34/VPS34 and the Vps15/VPS15 N-terminal myristoylation site. The other arm includes the C-terminal domains of Vps30/BECN1 and Atg14/ATG14L, corresponding to BARA and BATS domains, respectively ${ }^{95,98}$.
The crystal structure of Drosophila melanogaster Vps34 revealed a C-terminal loop region can interact with membranes and allow Vps34 activation ${ }^{93}$. The VPS34 Cterminus is regulated by acetylation at $\mathrm{K} 771$, which hinders the affinity of VPS34 for its substrate $\mathrm{PI}^{100}$. Aromatic residues in a surface loop of the BARA domain of Vps30/ BECLIN1 serve as a hydrophobic finger to mediate direct association with membranes ${ }^{94,95,101,102}$. VPS34 catalytic site geometry is strongly influenced by the presence of VPS15, indicating that VPS15 has a central role in scaffolding complex assembly ${ }^{103}$. Accordingly, it is thought that the PI3KC3-C1 complex is associated with membranes via the tips of the two arms of the PI3KC3-C1 complex carrying the Vps34/VPS34 C-terminus, the myristoylated Vps15/VPS15 N-terminus, the aromatic finger in Vps30/BECN1 and the ATG14L C-terminal 


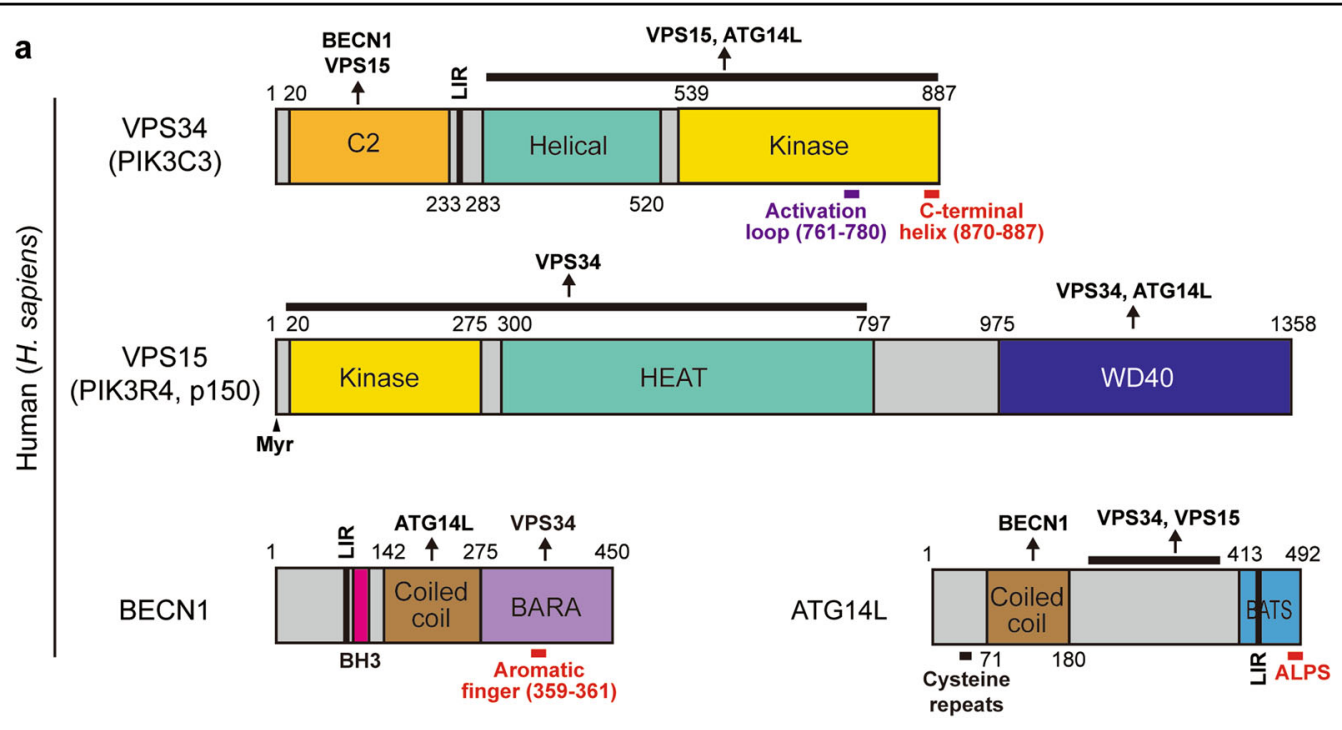

b

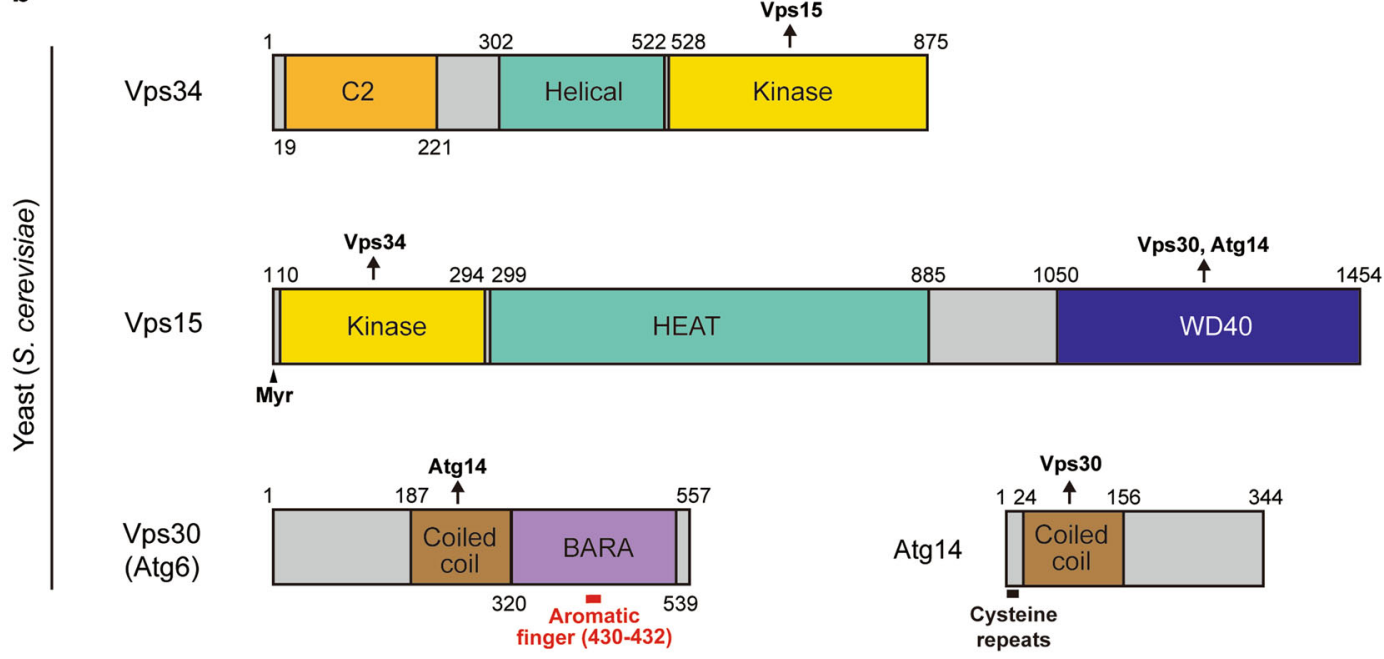

C

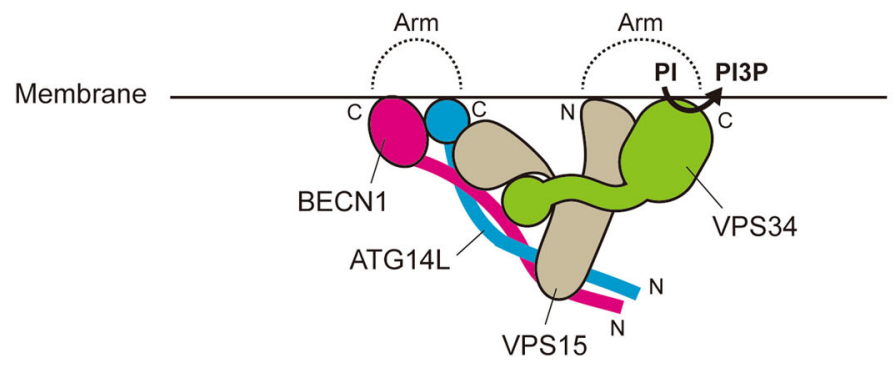

Fig. 4 The class III PI3K complex I (PI3KC3-C1) synthesizes PI3P at the autophagy initiation site. $\mathbf{a}$, $\mathbf{b}$ The domain structures of $H$. sapiens PI3KC3-C1 components (a) and S. cerevisiae PI3KC3-C1 components (b). c The proposed structure of mammalian PI3KC3-C1 complex. HEAT, Huntingtin, elongation factor 3, the PR65/A subunit of protein phosphatase 2A and the lipid kinase Tor; BH3 BCl-2 homology 3, LIR LC3-interacting region, BARA $\beta$-a repeated autophagy-specific, Myr Myristoylation, BATS BAKOR and ATG14L autophagosome-targeting sequence, ALPS amphipathic lipid packing sensor. 
BATS domain (Fig. 4c). The BATS domain binds small liposomes containing $\mathrm{PI} 3 \mathrm{P}$ or $\mathrm{PI}(4,5) \mathrm{P}_{2}$ and senses membrane curvature via an amphipathic helix loop (ALPS motif, Fig. 4a) ${ }^{96}$. Thus, the VPS34 C-terminus determines the orientation of the PI3KC3-C1 complex, while ATG14L1 BATS domain is critical to sense membrane curvature and mediate the lipid specificity to target membranes ${ }^{95,99}$.

The PI3KC3-C1 complex components also contain functional LIR motifs and interact preferentially with GABARAP and GABARAPL1 (Fig. 4a). As the LIR motif in ATG14L is in close apposition to its BATS domain, these two motifs might work as a coincidence detector for specific targeting of autophagic membranes ${ }^{104}$.

\section{Atg18/WIPI proteins: a transmitter of PI3P signals}

Atg18/Atg21/WIPI proteins belong to the PROPPINs ( $\beta$-propellers that bind polyphosphoinositides) family and work as PI3P effectors in autophagy. Atg21 is likely to be restricted to yeast and function mainly in selective autophagy $^{105,106}$. A major role of Atg18/WIPI proteins is to transmit PI3P signals to downstream ATG proteins (Fig. 5a). In yeast, Atg18 interacts with Atg2 and regulates PAS recruitment of Atg2 $2^{33,107}$. In mammals, the Atg18 homolog WIPI4 interacts with ATG2 proteins ${ }^{108-110}$. WIPI2B most prominently functions in LC3 lipidation in mammals ${ }^{111}$, via its unique interaction with ATG16L $1^{10}$. Thus, individual WIPI proteins have some preferences for their binding partners. This is the case also for C.elegans: the WIPI3/4 ortholog EPG-6 binds to ATG-2, while the WIPI1/2 ortholog ATG-18 does not ${ }^{112}$. As C. elegans atg18 and epg-6 mutants show different autophagic defects, it is thought that they act at distinct steps ${ }^{112}$. These functional differences of Atg18/WIPI proteins in autophagy are presumably caused by differences in their binding partners $^{10,109,112}$.

Atg18/WIPI proteins target to autophagic membranes by directly interacting with PI3P via a conserved FRRG motif (Fig. 5a, FRRG or LRRG) ${ }^{113,114}$. The PI3P binding of Atg18 proteins is required for full autophagic activity $^{107,115}$. Structural analyses have revealed that Atg18 proteins fold into a seven-bladed $\beta$-propeller and contain two PI3P binding pockets at blades 5 and 6 which are composed of two arginine residues located in the conserved FRRG motif ${ }^{16,117}$. As Atg2/ATG2-binding and Atg16L1-binding sites are located at the opposite surface from the FRRG motif ${ }^{10,108,110,118,119}$, this architecture enables Atg18/WIPIs to interact with PI3P and downstream ATG proteins simultaneously.

In addition, a hydrophobic loop in blade 6 serves as a membrane anchor by inserting deeply into the lipid bilayer (Fig. 5a, 6CD loop) ${ }^{117,120}$. In Komagataella phaffii (previously known as Pichia pastoris), PIPs binding of PpAtg18 is modulated by phosphorylation of the loop region ${ }^{121}$. Of note, this loop region of yeast Atg18 can fold into an amphipathic $\alpha$-helix, and is likely to be well conserved among Atg18/WIPI homologs ${ }^{122}$. The property of the loop might be one of main reasons why Atg18/ WIPI proteins preferentially bind to smaller liposomes in vitro ${ }^{120}$ and localize to the edge of the phagophore in vivo ${ }^{46}$. Thus, direct membrane association of Atg18/ WIPI proteins is controlled by two factors, membrane anchoring via the hydrophobic loop in blade 6 and PI3Pbinding via the FRRG motif ${ }^{17,120}$. Furthermore, Atg2Atg18 complex formation ${ }^{107,119,123}$ and Atg18/WIPI oligomerization $^{109,124}$ can further stabilize the membrane association of Atg18/WIPI proteins.

\section{Atg2/ATG2: a membrane tether and lipid transfer protein}

The precise role of Atg2/ATG2 in autophagy has recently been established. Single particle EM analyses have resolved the architecture of Atg2-Atg18 and ATG2A/B-WIPI4 complexes ${ }^{108,110}$. Atg2/ATG2 and Atg18/WIPI4 demonstrate a rod-shaped and a globular structure, respectively. Atg2 has membrane-binding regions at both ends of the rod structure (Fig. 5b, red lines), the length of which is about $\sim 200 \AA$ (Fig. $5 c)^{110,125,126}$, and can tether small liposomes in vitro ${ }^{110,126}$. WIPI4 is stably attached to one end of the ATG2 rod. In a complex with WIPI4, ATG2A can tether a PI3P-containing vesicle to another PI3P-free vesicle ${ }^{110}$. In line with this, high-resolution microscopy analyses have shown that Atg2/ATG2 localizes to the edge of the phagophore in close apposition to the ERES in yeast ${ }^{46,47}$ and contact sites between ER and autophagosomal membranes in mammalian cells ${ }^{127}$. The $\mathrm{N}$-terminal 46 residues of Atg2 localizes to the ER, and the membrane-binding region in the $\mathrm{C}$-terminal region is required for the targeting of the Atg2-Atg18 complex to the PAS ${ }^{126}$. Furthermore, both $\mathrm{N}$-terminal and $\mathrm{C}$-terminal regions of Atg2 are required to restore autophagy deficiency in $\operatorname{atg} 2 \triangle$ cells. Thus, Atg2/ATG2 serves as a tether for early autophagic structures to the ER membranes by collaborating with Atg18 in yeast and WIPI4 in mammals ${ }^{10,126}$. Yet, the contribution of WIPI4 is limited in mammals ${ }^{128,129}$. Instead, a functional LIR/GIM (LC3/ GABARAP-interacting motif) has been found in ATG2, which is indispensable for autophagy ${ }^{129}$. Atg9 also facilitates Atg2-dependent contact site formation in yeast ${ }^{130}$. Thus, multiple factors contribute to Atg2/ATG2-dependent tethering activity.

In addition to the tethering function of Atg2/ATG2, it possesses lipid transfer activity. Recently, the crystal structure of the $\mathrm{N}$-terminus of Atg2 has been solved revealing a tubular architecture with a hydrophobic cavity that can harbor tens of glycerophospholipids at once (Fig. $5 \mathrm{~b}$, green lines $)^{131}$, and can transfer phospholipids 
a

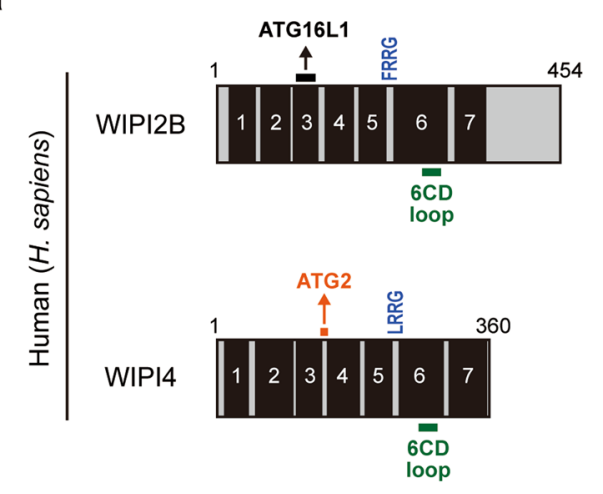

b

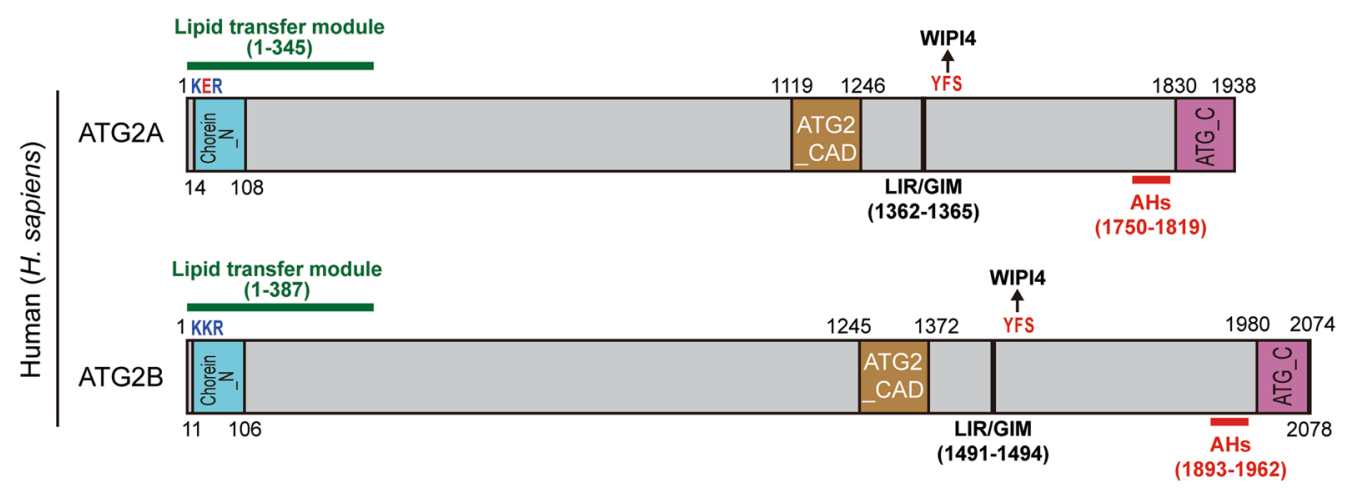

c

Acceptor membrane (The phagophore)

Donor membrane
(ER membrane)
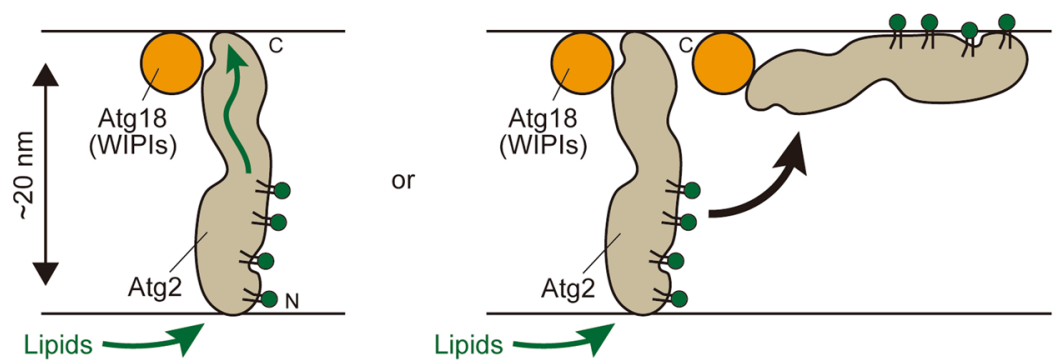

Fig. 5 Atg2/ATG2 functions as a membrane tether and lipid transfer protein in autophagy. a The domain structures of WIPI2B and WIPI4 in human (left) and Atg18 and Atg21 in S. cerevisiae (right). b The domain structures of Atg2/ATG2 proteins. c Models of Atg2/ATG2-dependent lipid transfer. Chorein_N N-terminal region of Chorein or VPS13, Atg2_CAD autophagy-related protein 2 CAD motif, ATG_C autophagy-related protein C-terminal domain, LIR/GIM LC3/GABARAP-interacting region, AH amphipathic helix.

with little head group specificity using in vitro liposome assays $^{127,131}$. The Atg2/ATG2-dependent lipid transfer depends on packing defects and negatively charged membranes ${ }^{123,131}$. In line with these in vitro data, the $\mathrm{N}$ terminal region of Atg2/ATG2 is essential for autophagic flux in both yeast and mammalian cells ${ }^{125-127,131}$. Surprisingly, overexpression of the Atg2 $\mathrm{N}$-terminus can restore autophagy deficiency in $A T G 2 A / B$ DKO cells ${ }^{127}$, suggesting that the tethering function of Atg2/ATG2 can be rescued by overexpression of the lipid transfer domain. 
Given that the Vps13 N-terminus, a homologous lipid transfer domain ${ }^{132}$, can be substituted for the corresponding region of Atg2 during autophagy ${ }^{131}$, the major function of Atg2/ATG2 in autophagy is lipid transport. How Atg2/ATG2 proteins accomplish unidirectional lipid transport and grow the phagophore membranes are key issues to be solved (Fig. 5c).

\section{Atg16/ATG16L1: a determinant of Atg8/LC3 family lipidation sites}

The Atg12-Atg5-Atg16/ATG12-ATG5-ATG16L1 complex is composed of the Atg12-Atg5/ATG12-ATG5 conjugate and a dimeric coiled-coil protein Atg16/ATG16L1. Atg12-Atg5/ATG12-ATG5 conjugate acts as an E3-like enzyme in the Atg8/LC3-PE conjugation reaction by facilitating the transfer of Atg8/LC3s/GABARAPs from an E2like Atg3/ATG3 to PE (Fig. 6a) ${ }^{133-136}$. Although Atg16/ ATG16L1 is dispensable for Atg8/LC3 lipidation reactions in vitro ${ }^{133,135}$, Atg16 can enhance Atg8 lipidation activity against low-curvature liposomes ${ }^{137}$ and immobilize Atg8$\mathrm{PE}$ and Atg12-Atg5 complexes on membranes in vitro ${ }^{138}$. In addition, Atg16/ATG16L1 has a key role in determining the site of Atg8/LC3 lipidation by controlling the targeting of the Atg12-Atg5/ATG12-ATG5 conjugate in vivo ${ }^{139}$.

The Atg12-Atg5-Atg16/ATG12-ATG5-ATG16L1 complex is recruited to PAS and the phagophore dependent on PI3P ${ }^{140-142}$. To date, several proteins have been reported as Atg16/Atg16L1-binding proteins (Fig. 6b). ATG16L1 interacts with Rab33B ${ }^{143}$, FIP200 ${ }^{144-146}$ and WIPI2B ${ }^{10}$ via distinct amino acid residues in a region between a coiled-coil domain and a WD-repeat domain in ATG16L1. Furthermore, a WD-repeat domain in the Cterminus of mammalian ATG16L1 interacts with ubiquitin ${ }^{146}$. Similar interactions have been reported in yeast. Atg21, an Atg18 paralog, binds to Atg16 ${ }^{147}$. The Atg16 complex associates with Atg1 complexes via the $\mathrm{N}$ terminal region of Atg12 ${ }^{148}$. Among these interactors, the ATG16L1-WIPI2B binding is the major PI3P-dependent interaction for membrane recruitment of ATG12-ATG5ATG16L1 complex in starvation-induced autophagy ${ }^{10}$.

More recently, ATG16L1 was found to have the ability to bind lipids. ATG16L1 has three membrane-binding domains, in the $\mathrm{N}$-terminal region, the coiled-coil domain $(C C D)$ and the $\beta$-isoform-specific region. The $\mathrm{N}$-terminal region, which contains an amphipathic helix (Fig. 6b, red lines), is universally required for the lipidation of LC3 family proteins, while the $\beta$-isoform-specific region (Fig. 6b, purple lines) is essential for VPS34independent LC3 lipidation at perturbed endosomes ${ }^{149}$, while the CCD has an intrinsic ability to bind lipids that is also required for LC3 lipidation (Fig. 6b, green lines) ${ }^{150}$. In summary, the Atg16/ATG16L1 complex is recruited to the target membranes through multiple-interacting partners, including both proteins and lipids. These dependencies can be changed in response to a physiological context ${ }^{149-151}$.

\section{Atg3/ATG3: an executor of Atg8/LC3 lipidation}

Atg3/ATG3 acts as the E2-like enzyme that catalyzes lipidation of Atg8/LC3 family proteins by localizing to the PAS and the phagophore (Fig. 6a) ${ }^{152-155}$. An amphipathic helix found in the N-terminus of Atg3 is critical for its association with membranes and Atg8/LC3 lipidation (Fig. 6c, red lines) ${ }^{156-158}$. This region is involved in the preferential association of Atg3/ATG3 with membranes containing high curvatures and/or conical lipids, such as $\mathrm{PE}^{156}$. On the other hand, lipidation of Atg8/LC3 family proteins is stimulated by acidic phospholipids in vitro ${ }^{29,158}$. This is probably due to the fact that membrane association of ATG3 relies on membrane charge in addition to lipid packing defects, which lead to cavities for the interaction of peripheral proteins ${ }^{158}$. Atg3 localization is also regulated by its interaction with Atg8 as a positive feedback to facilitate Atg8 lipidation ${ }^{155}$. In addition, acetylation at K19 and K48 of Atg3 significantly enhances the membrane association of Atg3 in the presence of physiological level of $\mathrm{PE}^{159,160}$. Overall, these multiple factors mediate Atg3 membrane targeting in vivo.

\section{The ER membrane: a platform for autophagosome formation}

The membrane origins and sources for the autophagosome remain under debate, as several membrane sources have been proposed in mammals, including the $\mathrm{ER}^{161-163}$, mitochondria ${ }^{164}$, the $\mathrm{PM}^{165}$, the ERGIC ${ }^{166}$, the recycling endosome $^{167,168}$, and lipid droplets (LD) ${ }^{169}$. Among these membrane sources it is becoming clear that the ERrelated membranes are the primary membrane source and serve as a platform for autophagosome formation. In support of evidence showing the contribution of the ER membranes, omegasomes have been well characterized as an autophagy-related ER structure (ER subdomain): it is a PI3P-enriched membrane labeled by DFCP1, formed in response to starvation and dynamically connected to the ER and the phagophores ${ }^{161}$. Membrane contacts between the ER and phagophore have been observed by electron microscopy. Rough ER membranes are attached to both the outer and inner surfaces of cup-shaped phagophores $^{162,163}$. Correlative light and electron microscopy (CLEM) and correlative cryo-fluorescence and cryo-soft $\mathrm{X}$-ray microscopy (cryo-CLXM) have shown that omegasomes are composed of clusters of tubular/vesicular elements, part of which are continuous with the phagophores and/or the ER membranes ${ }^{170,171}$. These ER subdomains may also form organelle contact sites between the ER and other compartments, such as the mitochondria $^{172}, \mathrm{LDs}^{173}$, and the $\mathrm{PM}^{174}$. It has been also shown that the edge regions of phagophore membranes are attached 


\section{a}

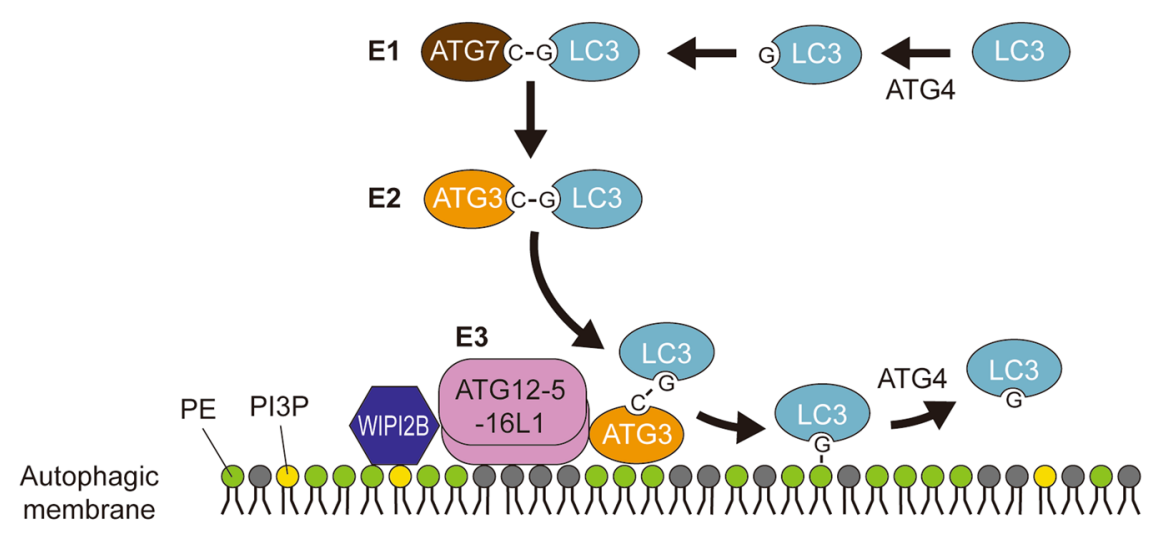

b

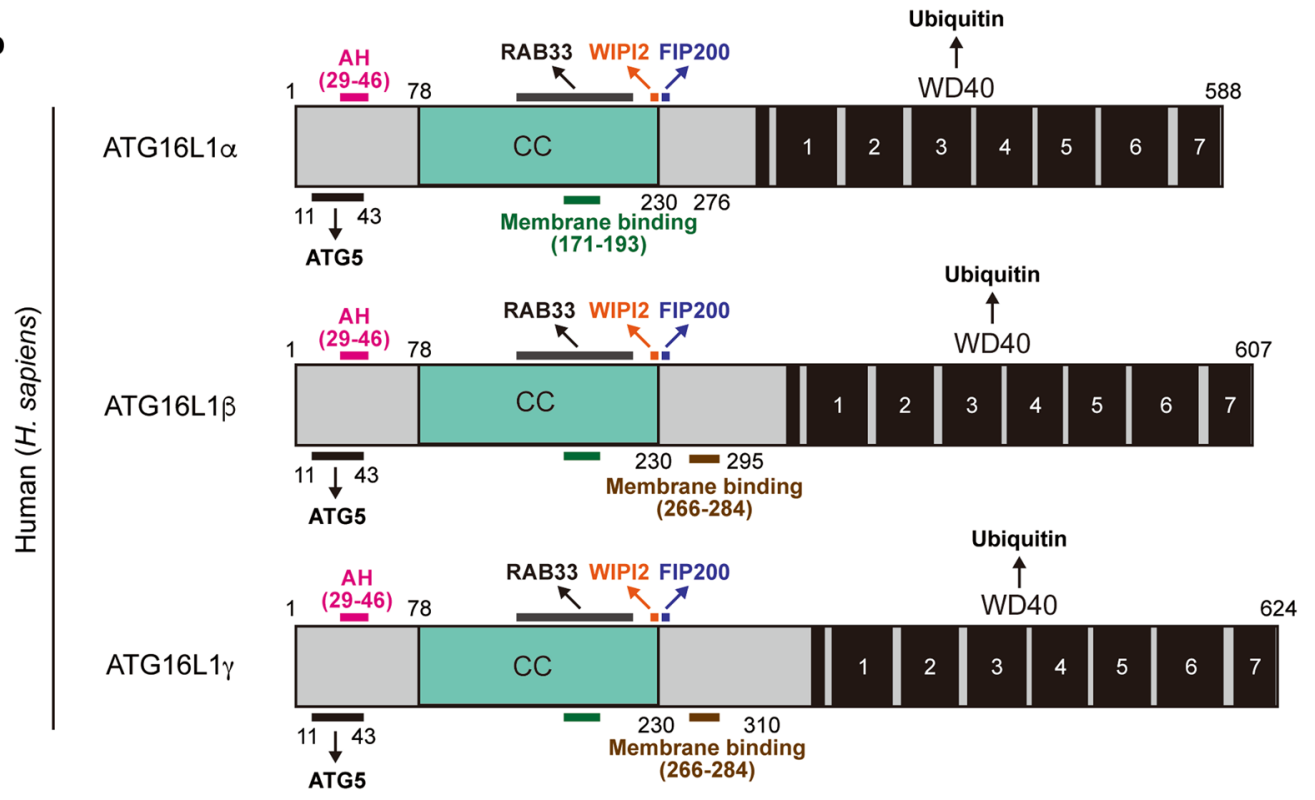

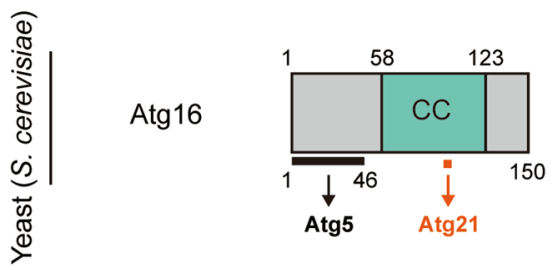
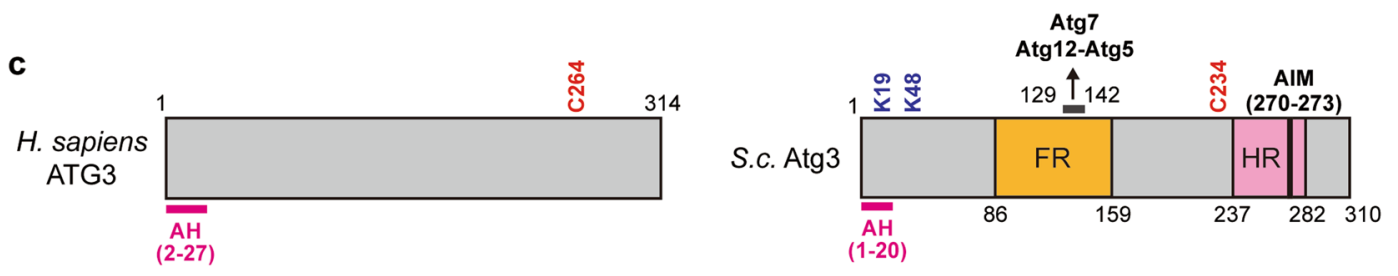

Fig. 6 Atg12-5-16/ATG12-5-16L1 and Atg3/ATG3 catalyze lipidation of Atg8/LC3 family proteins. a The lipidation system of LC3. ATG4 cleaves the C-terminal residues of LC3 to expose glycine (G) residue. Then, LC3 is activated by ATG7 (E1 enzyme) and transferred to ATG3 (E2 enzyme). ATG12-5-16L1 complex facilitates the transfer of LC3 from ATG3 to PE. A PI3P-binding protein WIPI2B controls membrane recruitment of ATG12-5-16L1 complex under starvation condition. b The domain structures of H. sapiens ATG16L1 and S. cerevisiae Atg16 proteins. c The domain structures of $\mathrm{H}$. sapiens ATG3 and S. cerevisiae Atg3 proteins. CC coiled-coil, AH amphipathic helix, FR flexible region, HR handle region, AIM Atg8 family-interacting motif. 
to the ERES ${ }^{46-48,175}$ and that COPII coat subunits are required for autophagosome formation ${ }^{45,176,177}$ and a COPII cargo protein is incorporated into autophagic membranes ${ }^{178}$, suggesting ER-derived COPII vesicles could contribute to autophagic membranes.

ATG proteins are sequentially recruited to the ER and autophagic membranes in response to autophagy induction (Fig. 1b, c). Their localization is changed depending on different stages of autophagosome formation. In mammalian cells, the ULK complex is first recruited to the ER membrane to initiate autophagy. At middle and late stages of autophagosome formation, the ULK complex also localizes to the omegasome, the phagophore and autophagosome $\mathrm{e}^{32,34}$. ATG14L, a component of mammalian PI3KC3-C1, targets to both the ER-related membranes and phagophore to generate PI3P ${ }^{96,97,172}$, while the lipid transfer protein ATG2 is specifically localized to the ER and phagophore contact sites $^{127}$. The unique localization of ATG2 presumably reflects its dual membrane targeting and tethering function. WIPI2B and ATG16L1 are mainly localized to the phagophore dependently on PI3P under starvation condition ${ }^{10}$. Given that it has been proposed that the ERGIC is a key membrane source for LC3 lipidation $^{166}$, ATG12-5-16L1 and ATG3 are thought to be recruited to the ERGIC in addition to the phagophore membrane to execute LC3 lipidation. Similarly, yeast Atg proteins are also distributed to distinct compartments during autophagosome formation. Atg1 complex, Atg14, Atg12-5-16 complex, and Atg3 localize to the phagophore $46,47,154,155$, while Atg2, Atg18, and Atg9 are enriched at the edge regions of phagophore membranes, which is close apposition to the ERES $^{46,47,130}$. On the other hand, there are some differences between yeast and mammalian cells. There are no counterparts to ERGIC and the omegasomes in yeast, but instead the phagophore is formed in the vicinity of the ERES and the vacuole ${ }^{46,47,130}$. Some Atg proteins such as Atg13, Atg17, and Atg14 are distributed to the vacuole and phagophore contact sites ${ }^{46}$. Further analyses are needed to reveal how the complex membrane targeting of Atg proteins is achieved in yeast and mammalian cells.

Membrane recruitment of the ULK complex precedes omegasome formation in mammalian cells. In highresolution analysis using super-resolution microscopy, the initiation of autophagosome formation occurs in regions of the ER, where the ULK complex and the ATG9A vesicles are associated ${ }^{58}$. Interestingly, ERlocalized phospholipid synthesizing enzymes, such as PI synthase and PS synthase, are enriched in close proximity to the autophagosome initiation site ${ }^{34}$, implying a close relationship between phospholipid synthesis and autophagosome formation. The enrichment of these enzymes might facilitate PI3P generation and ATG2-dependent lipid transfer.

\section{VMP1 and TMEM41B: a key regulator of ion homeostasis during autophagosome formation?}

VMP1 (also known as TMEM49) is a multi-spanning membrane protein localized at the ER and required for autophagosome formation. The VMP1 gene is absent in yeast, but conserved in most higher eukaryotes. Given its ER localization, VMP1 has been thought to be a key player in an ER-related event essential for autophagosome formation. Although VMP1-GFP puncta have been observed after overexpression, this puncta formation is not essential for the autophagy function of VMP1 ${ }^{179,180}$. Impaired VMP1 function causes not only accumulation of abnormal autophagic structures ${ }^{181,182}$, but also pleiotropic effects, such as protein secretion defects ${ }^{183,184}$, impaired lipoprotein secretion ${ }^{185}$, abnormal distribution of PI4P and phospholipid metabolizing enzymes ${ }^{186}$, accumulation of lipid droplets ${ }^{179,187}$, and enhanced ER-organelle contact sites ${ }^{179,187}$. Accordingly, VMP1 function is not limited to autophagy, and the molecular mechanism underlying the abnormalities of VMP1-deficient cells remains unclear.

A recent study has revealed a new functional link between VMP1 and a calcium pump SERCA (sarcoendoplasmic reticulum calcium transport ATPase). VMP1 interacts with SERCAs that transport cytosolic $\mathrm{Ca}^{2+}$ into the ER lumen and positively regulates their $\mathrm{Ca}^{2}$ ${ }^{+}$-ATPase activity, suggesting that VMP1 coordinates multiple organelle contact sites by maintaining local $\mathrm{Ca}^{2}$ ${ }^{+}$levels via SERCA activity regulation ${ }^{179}$. More detail about VMP1 function has been provided by studies on $T M E M 41 B$, a newly identified autophagy-related gene in genome-wide CRISPR screens ${ }^{180,188,189}$. Interestingly, TMEM41B localizes to the ER and shares a similar protein structure with VMP1. As TMEM41B and VMP1 form a complex, and overexpression of VMP1 restores impaired autophagic flux in TMEM41B KO cells, it has been proposed that they may be half-transporters and function together in autophagy as a full transporter by forming a complex. More work is needed to support this exciting hypothesis and the role of VMP1 and TMEM41B in ion homeostasis during autophagosome formation.

\section{Emerging roles of lipid metabolism in autophagosome biogenesis}

There is accumulating evidence that autophagy can be regulated by sphingolipids ${ }^{190-192}$. Treatment with a shortchain ceramide (C2-ceramide) is able to induce autophagy in various different cell lines, such as human cervical cancer cells, colon cancer cells, and malignant glioma cells $^{193-197}$. Consistent with these reports, de novo 
synthesis of ceramide is required for the induction of autophagy in stimulated RAW264.7 macrophage cells ${ }^{198}$ and the mouse liver ${ }^{199}$, as well as in yeast ${ }^{200}$. The proautophagic effects by ceramide were proposed to be caused by upregulation of BECN1 activity and/or interfering with class I PI3K/Akt signaling pathway ${ }^{194,197}$, yet the molecular details remain to be elucidated.

Sphingosine 1-phosphate (S1P) is a simple lysophospholipid known to promote cell survival. Recently, the diverse roles of S1P-metabolizing enzymes in autophagy are being discovered. Overexpression of sphingosine kinase 1 (SK1), which generates S1P from sphingosine, stimulates autophagy in MCF-7 cells and primary neurons $^{201,202}$. Depletion of S1P phosphohydrolase 1, which mediates degradation of S1P by dephosphorylation, results in the induction of autophagy, suggesting that accumulation of S1P can promote autophagy ${ }^{203}$. S1P not only binds to S1P receptors at the cell surface ${ }^{204,205}$, but also acts on intracellular membranes ${ }^{202,203}$, but how intracellular S1P works on autophagy remains unclear. Notably, S1P is cleaved by SGPL1 (sphingosine phosphate lyase 1) into hexadecenal and ethanolamine phosphate, which can be consumed for the synthesis of PE. Given that autophagosome formation is compromised in SGPL1-deficient brains ${ }^{206}$, PE generated from the S1P degradation products might play a key role in autophagy in neurons. Sphingomyelin is also involved in autophagy. Overloading of sphingomyelin affects ATG9A transport and induces accumulation of immature autophagosomes $^{207}$. The increase of ceramide 1-phosphate (C1P) at the Golgi induced by CPTP (C1P transfer protein) knockdown also affects ATG9A distribution ${ }^{208}$. Neutral sphingomyelinase 2 induces autophagy by increasing ceramide levels in the Golgi ${ }^{209}$.

The effect of fatty acids on autophagy has drawn some attention in recent years. Saturated fatty acid palmitate induces autophagy in several cell lines ${ }^{210-212}$, although it has an inhibitory effect in different experimental conditions $^{213}$. Mono-unsaturated (oleate) and $\omega-6$ polyunsaturated fatty acids (arachidonic acid) also activate autophagy ${ }^{214-216}$. Yet, it is reported that the underlying mechanisms of saturated fatty acids- and monounsaturated fatty acids-induced autophagy are not the same $^{216}$. On the other hand, stearoyl-CoA desaturase, an enzyme generating mono-unsaturated fatty acids, is indispensable for efficient autophagosome formation ${ }^{217-219}$. Therefore, the synthetic pathway of mono-unsaturated fatty acids also contributes to autophagy. Finally, a recent study has shown that trans-unsaturated fatty acids inhibit autophagy induced by saturated fatty acids ${ }^{220}$, implying complicated connections between autophagic effects caused by different types of fatty acids. Further analyses are needed to elucidate how individual fatty acids act on autophagy.
Autophagosome formation can be affected by changes in phospholipid metabolism. PLD1 hydrolyzes phosphatidylcholine (PC) to generate phosphatidic acid (PA). In mammalian cells, PLD1 localizes to autophagosomerelated structures and facilitates autophagy $221-223$. It has been proposed that its product PA plays a key role in autophagosome formation ${ }^{221,224}$ and/or lysosomal functions $^{223}$. However, contrary to these findings, another study has shown that the inhibition of PLD1 results in an enhancement of autophagic flux ${ }^{225}$. Therefore, PLD1 might function as both positive and negative regulator of autophagy in a context-dependent manner. On the other hand, a role for lipid droplets (LDs) in autophagosome biogenesis has been reported. LDs are made of a hydrophobic core of neutral lipids and a surrounding phospholipid monolayer. In mammalian cells, a neutral lipase PNPLA5 positively regulates autophagy ${ }^{169}$. In line with this study, autophagy is impaired upon nitrogen starvation in LD-deficient yeast mutant ${ }^{173,226}$. Interestingly, a recent study has found that the autophagy deficiency in the LDdeficient yeast mutant can be improved by suppressing fatty acid synthesis or by restoring the altered phospholipid composition, indicating that LDs are not necessarily essential for autophagosome biogenesis, and that LDs play a key role in autophagy regulation by relieving metabolic stress caused by excess fatty acid synthesis and altered phospholipid composition ${ }^{227}$.

\section{Concluding remarks}

Thanks to the tremendous development of novel techniques, our knowledge of the molecular machinery underlying autophagosome formation has been expanded. Of note, accumulating evidence suggest that ATG proteins are directly involved in membrane lipid dynamics and organization during autophagy. While these findings have pushed the field forward, our current knowledge of lipid composition and distribution in autophagic membranes is very limited ${ }^{228}$. Therefore, advanced techniques to detect and evaluate lipid distribution and changes in lipid composition in vivo need to be further developed $^{229,230}$. In vitro reconstitution ${ }^{231}$, in silico simulation $^{232}$ and theoretical analysis ${ }^{233}$ are also required to obtain deeper insights into the relationship between ATG proteins and membrane lipids. In summary, it will be important to reveal how ATG proteins organize membrane lipids for understanding the detailed mechanisms of autophagosome biogenesis.

\section{Acknowledgements}

We thank Hayashi Yamamoto and members of the Tooze lab, especially Harold Jefferies, Javier Hervas, and Wenxin Zhang, for helpful discussions. This project has received funding from the European Research Council (ERC) under the European Union's Horizon 2020 research and innovation program (grant agreement No 788708) and the Francis Crick Institute, which receives its core funding from Cancer Research UK (FC001187); the UK Medical Research Council (FC001187); and the Welcome Trust (FC001187). 


\section{Author contributions}

T.N. and S.A.T. wrote the manuscript.

\section{Conflict of interest}

The authors declare that they have no conflict of interest.

\section{Publisher's note}

Springer Nature remains neutral with regard to jurisdictional claims in published maps and institutional affiliations.

Received: 3 December 2019 Accepted: 21 March 2020

Published online: 26 May 2020

\section{References}

1. Dunn, W. A. Jr. Studies on the mechanisms of autophagy: formation of the autophagic vacuole. J. Cell Biol. 110, 1923-1933 (1990).

2. Deter, R. L., Baudhuin, P. \& De Duve, C. Participation of lysosomes in cellular autophagy induced in rat liver by glucagon. J. Cell Biol. 35, C11-C16 (1967)

3. Novikoff, A. B. \& Shin, W. Y. Endoplasmic reticulum and autophagy in rat hepatocytes. Proc. Natl Acad. Sci. USA 75, 5039-5042 (1978).

4. Kopitz, J., Kisen, G. O., Gordon, P. B., Bohley, P. \& Seglen, P. O. Nonselective autophagy of cytosolic enzymes by isolated rat hepatocytes. J. Cell Biol. 111, 941-953 (1990).

5. Stromhaug, P. E., Berg, T. O., Fengsrud, M. \& Seglen, P. O. Purification and characterization of autophagosomes from rat hepatocytes. Biochem. J. 335 (Pt 2), 217-224 (1998).

6. Takeshige, K., Baba, M., Tsuboi, S., Noda, T. \& Ohsumi, Y. Autophagy in yeast demonstrated with proteinase-deficient mutants and conditions for its induction. J. Cell Biol. 119, 301-311 (1992).

7. Tsukada, M. \& Ohsumi, Y. Isolation and characterization of autophagydefective mutants of Saccharomyces cerevisiae. FEBS Lett. 333, 169-174 (1993).

8. Harding, T. M., Morano, K. A., Scott, S. V. \& Klionsky, D. J. Isolation and characterization of yeast mutants in the cytoplasm to vacuole protein targeting pathway. J. Cell Biol. 131, 591-602 (1995).

9. Thumm, M. et al. Isolation of autophagocytosis mutants of Saccharomyces cerevisiae. FEBS Lett. 349, 275-280 (1994).

10. Dooley, H. C. et al. WIPI2 links LC3 conjugation with PI3P, autophagosome formation, and pathogen clearance by recruiting Atg12-5-16L1. Mol. Cell 55, 238-252 (2014).

11. Nakatogawa, H., Suzuki, K., Kamada, Y. \& Ohsumi, Y. Dynamics and diversity in autophagy mechanisms: lessons from yeast. Nat. Rev. Mol. Cell Biol. 10 458-467 (2009).

12. Lamb, C. A., Yoshimori, T. \& Tooze, S. A. The autophagosome: origins unknown, biogenesis complex. Nat. Rev. Mol. Cell Biol. 14, 759-774 (2013).

13. Liang, $X$. H. et al. Induction of autophagy and inhibition of tumorigenesis by beclin 1. Nature 402, 672-676 (1999).

14. Kuma, A. et al. The role of autophagy during the early neonatal starvation period. Nature 432, 1032-1036 (2004).

15. Tsukamoto, S. et al. Autophagy is essential for preimplantation development of mouse embryos. Science 321, 117-120 (2008).

16. Singh, R. et al. Autophagy regulates lipid metabolism. Nature 458, 1131-1135 (2009).

17. Nakagawa, I. et al. Autophagy defends cells against invading group A Streptococcus. Science 306, 1037-1040 (2004).

18. Hara, T. et al. Suppression of basal autophagy in neural cells causes neurodegenerative disease in mice. Nature 441, 885-889 (2006).

19. Komatsu, M. et al. Loss of autophagy in the central nervous system causes neurodegeneration in mice. Nature 441, 880-884 (2006).

20. Morishita, H. \& Mizushima, N. Diverse cellular roles of autophagy. Annu. Rev. Cell. Dev. Biol. 35, 453-475 (2019).

21. Seglen, P. O. \& Gordon, P. B. 3-Methyladenine: specific inhibitor of autophagic/lysosomal protein degradation in isolated rat hepatocytes. Proc. Natl Acad. Sci. USA 79, 1889-1892 (1982).

22. Blommaart, E. F., Krause, U., Schellens, J. P., Vreeling-Sindelarova, H. \& Meijer, A J. The phosphatidylinositol 3-kinase inhibitors wortmannin and LY294002 inhibit autophagy in isolated rat hepatocytes. Eur. J. Biochem. 243, 240-246 (1997).

23. Petiot, A., Ogier-Denis, E., Blommaart, E. F., Meijer, A. J. \& Codogno, P. Distinct classes of phosphatidylinositol 3'-kinases are involved in signaling pathways that control macroautophagy in HT-29 cells. J. Biol. Chem. 275, 992-998 (2000).

24. Schu, P. V. et al. Phosphatidylinositol 3-kinase encoded by yeast VPS34 gene essential for protein sorting. Science 260, 88-91 (1993).

25. Volinia, S. et al. A human phosphatidylinositol 3-kinase complex related to the yeast Vps34p-Vps15p protein sorting system. EMBO J. 14, 3339-3348 (1995).

26. Kihara, A., Noda, T., Ishihara, N. \& Ohsumi, Y. Two distinct Vps34 phosphatidylinositol 3-kinase complexes function in autophagy and carboxypeptidase Y sorting in Saccharomyces cerevisiae. J. Cell Biol. 152, 519-530 (2001).

27. Mizushima, N., Yoshimori, T. \& Ohsumi, Y. The role of Atg proteins in autophagosome formation. Annu. Rev. Cell. Dev. Biol. 27, 107-132 (2011).

28. Sou, Y. S., Tanida, I., Komatsu, M., Ueno, T. \& Kominami, E. Phosphatidylserine in addition to phosphatidylethanolamine is an in vitro target of the mammalian Atg8 modifiers, LC3, GABARAP, and GATE-16. J. Biol. Chem. 281 3017-3024 (2006).

29. Oh-oka, K, Nakatogawa, H. \& Ohsumi, Y. Physiological pH and acidic phospholipids contribute to substrate specificity in lipidation of Atg8. J. Biol. Chem 283, 21847-21852 (2008).

30. Mercer, T. J., Gubas, A. \& Tooze, S. A. A molecular perspective of mammalian autophagosome biogenesis. J. Biol. Chem. 293, 5386-5395 (2018).

31. Papinski, D. \& Kraft, C. Regulation of autophagy by signaling through the Atg1/ULK1 complex. J. Mol. Biol. 428, 1725-1741 (2016).

32. Koyama-Honda, I., Itakura, E., Fujiwara, T. K. \& Mizushima, N. Temporal analysis of recruitment of mammalian ATG proteins to the autophagosome formation site. Autophagy 9, 1491-1499 (2013).

33. Suzuki, K., Kubota, Y., Sekito, T. \& Ohsumi, Y. Hierarchy of Atg proteins in preautophagosomal structure organization. Genes Cells 12, 209-218 (2007).

34. Nishimura, T. et al. Autophagosome formation is initiated at phosphatidylinositol synthase-enriched ER subdomains. EMBO J. 36, 1719-1735 (2017).

35. Itakura, E. \& Mizushima, N. Characterization of autophagosome formation site by a hierarchical analysis of mammalian Atg proteins. Autophagy 6, 764-776 (2010).

36. Karanasios, E. et al. Dynamic association of the ULK1 complex with omegasomes during autophagy induction. J. Cell Sci. 126, 5224-5238 (2013).

37. Chan, E. Y., Longatti, A., McKnight, N. C. \& Tooze, S. A. Kinase-inactivated ULK proteins inhibit autophagy via their conserved C-terminal domains using an Atg13-independent mechanism. Mol. Cell Biol. 29, 157-171 (2009).

38. Ragusa, M. J., Stanley, R. E. \& Hurley, J. H. Architecture of the Atg17 complex as a scaffold for autophagosome biogenesis. Cell 151, 1501-1512 (2012).

39. Rao, Y., Perna, M. G., Hofmann, B., Beier, V. \& Wollert, T. The Atg1-kinase complex tethers Atg9-vesicles to initiate autophagy. Nat. Commun. 7, 10338 (2016).

40. Fujioka, $Y$. et al. Structural basis of starvation-induced assembly of the autophagy initiation complex. Nat. Struct. Mol. Biol. 21, 513-521 (2014).

41. Stjepanovic, G. et al. Assembly and dynamics of the autophagy-initiating Atg1 complex. Proc. Natl Acad. Sci. USA 111, 12793-12798 (2014).

42. Lin, M. G., Schoneberg, J., Davies, C. W., Ren, X. \& Hurley, J. H. The dynamic Atg13-free conformation of the Atg1 EAT domain is required for phagophore expansion. Mol. Biol. Cell 29, 1228-1237 (2018).

43. Gatica, D. et al. The carboxy terminus of yeast Atg13 binds phospholipid membrane via motifs that overlap with the Vac8-interacting domain. Autophagy, in press.

44. Wang, J. et al. Ypt1 recruits the Atg1 kinase to the preautophagosomal structure. Proc. Natl Acad. Sci. USA 110, 9800-9805 (2013).

45. Ishihara, N. et al. Autophagosome requires specific early Sec proteins for its formation and NSF/SNARE for vacuolar fusion. Mol. Biol. Cell 12, 3690-3702 (2001).

46. Suzuki, K., Akioka, M., Kondo-Kakuta, C., Yamamoto, H. \& Ohsumi, Y. Fine mapping of autophagy-related proteins during autophagosome formation in Saccharomyces cerevisiae. J. Cell Sci. 126, 2534-2544 (2013).

47. Graef, M., Friedman, J. R., Graham, C., Babu, M. \& Nunnari, J. ER exit sites are physical and functional core autophagosome biogenesis components. Mol. Biol. Cell 24, 2918-2931 (2013).

48. Ge, L. et al. Remodeling of ER-exit sites initiates a membrane supply pathway for autophagosome biogenesis. EMBO Rep. 18, 1586-1603 (2017). 
49. Zhao, Y. G. et al. The ER contact proteins VAPAB interact with multiple autophagy proteins to modulate autophagosome biogenesis. Curr. Biol. 28, 1234-1245 (2018). e1234.

50. Murphy, S. E. \& Levine, T. P. VAP, a versatile access point for the endoplasmic reticulum: review and analysis of FFAT-like motifs in the VAPome. Biochim. Biophys. Acta 1861, 952-961 (2016).

51. Alemu, E. A. et al. ATG8 family proteins act as scaffolds for assembly of the ULK complex: sequence requirements for LC3-interacting region (LIR) motifs. J. Biol. Chem. 287, 39275-39290 (2012).

52. Kraft, C. et al. Binding of the Atg1/ULK1 kinase to the ubiquitin-like protein Atg8 regulates autophagy. EMBO J. 31, 3691-3703 (2012).

53. Nakatogawa, $\mathrm{H}$. et al. The autophagy-related protein kinase Atg1 interacts with the ubiquitin-like protein Atg8 via the Atg8 family interacting motif to facilitate autophagosome formation. J. Biol. Chem. 287, 28503-28507 (2012)

54. Mari, M. et al. An Atg9-containing compartment that functions in the early steps of autophagosome biogenesis. J. Cell Biol. 190, 1005-1022 (2010).

55. Yamamoto, $\mathrm{H}$. et al. Atg9 vesicles are an important membrane source during early steps of autophagosome formation. J. Cell Biol. 198 219-233 (2012)

56. Orsi, A. et al. Dynamic and transient interactions of Atg9 with autophagosomes, but not membrane integration, are required for autophagy. Mol. Biol. Cell 23, 1860-1873 (2012)

57. Longatti, A. et al. TBC1D14 regulates autophagosome formation via Rab11and ULK1-positive recycling endosomes. J. Cell Biol. 197, 659-675 (2012).

58. Karanasios, E. et al. Autophagy initiation by ULK complex assembly on ER tubulovesicular regions marked by ATG9 vesicles. Nat. Commun. 7, 12420 (2016).

59. Sekito, T., Kawamata, T., Ichikawa, R., Suzuki, K. \& Ohsumi, Y. Atg17 recruits Atg9 to organize the pre-autophagosomal structure. Genes Cells 14, 525-538 (2009).

60. Suzuki, S. W. et al. Atg13 HORMA domain recruits Atg9 vesicles during autophagosome formation. Proc. Natl Acad. Sci. USA 112, 3350-3355 (2015)

61. Papinski, D. et al. Early steps in autophagy depend on direct phosphorylation of Atg9 by the Atg1 kinase. Mol. Cell 53, 471-483 (2014).

62. Young, A. R. et al. Starvation and ULK1-dependent cycling of mammalian Atg9 between the TGN and endosomes. J. Cell Sci. 119, 3888-3900 (2006).

63. Zhou, C. et al. Regulation of MATG9 trafficking by Src- and ULK1-mediated phosphorylation in basal and starvation-induced autophagy. Cell Res. 27, 184-201 (2017).

64. Imai, K. et al. Atg9A trafficking through the recycling endosomes is required for autophagosome formation. J. Cell Sci. 129, 3781-3791 (2016).

65. Mattera, R., Park, S. Y., De Pace, R., Guardia, C. M. \& Bonifacino, J. S. AP-4 mediates export of ATG9A from the trans-Golgi network to promote autophagosome formation. Proc. Natl Acad. Sci. USA 114, E10697-E10706 (2017).

66. Popovic, D. \& Dikic, I. TBC1D5 and the AP2 complex regulate ATG9 trafficking and initiation of autophagy. EMBO Rep. 15, 392-401 (2014).

67. Davies, A. K. et al. AP-4 vesicles contribute to spatial control of autophagy via RUSC-dependent peripheral delivery of ATG9A. Nat. Commun. 9, 3958 (2018).

68. De Pace, R. et al. Altered distribution of ATG9A and accumulation of axonal aggregates in neurons from a mouse model of AP-4 deficiency syndrome. PLoS Genet. 14, e1007363 (2018).

69. Ivankovic, D. et al. Axonal autophagosome maturation defect through failure of ATG9A sorting underpins pathology in AP-4 deficiency syndrome. Autophagy 16, 391-407 (2020).

70. Backues, S. K. et al. Atg23 and Atg27 act at the early stages of Atg9 trafficking in S. cerevisiae. Traffic 16, 172-190 (2015).

71. Lipatova, Z. et al. Regulation of selective autophagy onset by a Ypt/Rab GTPase module. Proc. Natl Acad. Sci. USA 109, 6981-6986 (2012).

72. Kakuta, S. et al. Atg9 vesicles recruit vesicle-tethering proteins Trs 85 and Ypt1 to the autophagosome formation site. J. Biol. Chem. 287, 44261-44269 (2012)

73. Lamb, C. A. et al. TBC1D14 regulates autophagy via the TRAPP complex and ATG9 traffic. EMBO J. 35, 281-301 (2016).

74. Kakuta, S. et al. Small GTPase Rab1B is associated with ATG9A vesicles and regulates autophagosome formation. FASEB J. 31, 3757-3773 (2017).

75. Shirahama-Noda, K., Kira, S., Yoshimori, T. \& Noda, T. TRAPPIII is responsible for vesicular transport from early endosomes to Golgi, facilitating Atg9 cycling in autophagy. J. Cell Sci. 126, 4963-4973 (2013).
76. Zou, S. et al. Trs130 participates in autophagy through GTPases Ypt31/32 in Saccharomyces cerevisiae. Traffic 14, 233-246 (2013).

77. Soreng, $K$. et al. SNX18 regulates ATG9A trafficking from recycling endosomes by recruiting Dynamin-2. EMBO Rep. 19, e44837 (2018).

78. Takahashi, Y. et al. The Bif-1-Dynamin 2 membrane fission machinery regulates Atg9-containing vesicle generation at the Rab11-positive reservoirs. Oncotarget 7, 20855-20868 (2016).

79. Webber, J. L. \& Tooze, S. A. Coordinated regulation of autophagy by p38alpha MAPK through mAtg9 and p38IP. EMBO J. 29, 27-40 (2010).

80. Wang, K. et al. Phosphatidylinositol 4-kinases are required for autophagic membrane trafficking. J. Biol. Chem. 287, 37964-37972 (2012).

81. Judith, D. et al. ATG9A shapes the forming autophagosome through Arfaptin 2 and phosphatidylinositol 4-kinase IIIbeta. J. Cell Biol. 218, 1634-1652 (2019).

82. Wang, $H$. et al. GABARAPs regulate PI4P-dependent autophagosome:lysosome fusion. Proc. Natl Acad. Sci. USA 112, 7015-7020 (2015).

83. Lai, L. T. F. et al. Subnanometer resolution cryo-EM structure of Arabidopsis thaliana ATG9. Autophagy 16, 575-583 (2020).

84. Itakura, E., Kishi, C., Inoue, K. \& Mizushima, N. Beclin 1 forms two distinct phosphatidylinositol 3-kinase complexes with mammalian Atg14 and UVRAG. Mol. Biol. Cell 19, 5360-5372 (2008).

85. Sun, Q. et al. Identification of Barkor as a mammalian autophagy-specific factor for Beclin 1 and class III phosphatidylinositol 3-kinase. Proc. Natl Acad. Sci. USA 105, 19211-19216 (2008)

86. Matsunaga, K. et al. Two Beclin 1-binding proteins, Atg14L and Rubicon, reciprocally regulate autophagy at different stages. Nat. Cell Biol. 11, 385-396 (2009).

87. Araki, Y. et al. Atg38 is required for autophagy-specific phosphatidylinositol 3kinase complex integrity. J. Cell Biol. 203, 299-313 (2013).

88. Cao, Y. et al. NRBF2 regulates macroautophagy as a component of Vps34 Complex I. Biochem. J. 461, 315-322 (2014).

89. Lu, J. et al. NRBF2 regulates autophagy and prevents liver injury by modulating Atg14L-linked phosphatidylinositol-3 kinase III activity. Nat. Commun. 5, 3920 (2014)

90. Zhong, Y. et al. Nrbf2 protein suppresses autophagy by modulating Atg14L protein-containing Beclin 1-Vps34 complex architecture and reducing intracellular phosphatidylinositol-3 phosphate levels. J. Biol. Chem. 289, 26021-26037 (2014).

91. Ohashi, Y. et al. Characterization of Atg38 and NRBF2, a fifth subunit of the autophagic Vps34/PIK3C3 complex. Autophagy 12, 2129-2144 (2016).

92. Young, L. N., Cho, K., Lawrence, R., Zoncu, R. \& Hurley, J. H. Dynamics and architecture of the NRBF2-containing phosphatidylinositol 3-kinase complex of autophagy. Proc. Natl Acad. Sci. USA 113, 8224-8229 (2016).

93. Miller, S. et al. Shaping development of autophagy inhibitors with the structure of the lipid kinase Vps34. Science 327, 1638-1642 (2010).

94. Huang, W. et al. Crystal structure and biochemical analyses reveal Beclin 1 as a novel membrane binding protein. Cell Res. 22, 473-489 (2012)

95. Rostislavleva, $\mathrm{K}$. et al. Structure and flexibility of the endosomal Vps34 complex reveals the basis of its function on membranes. Science $\mathbf{3 5 0}$, aac7365 (2015).

96. Fan, W., Nassiri, A. \& Zhong, Q. Autophagosome targeting and membrane curvature sensing by Barkor/Atg14(L). Proc. Natl Acad. Sci. USA 108, 7769-7774 (2011).

97. Matsunaga, K. et al. Autophagy requires endoplasmic reticulum targeting of the PI3-kinase complex via Atg14L. J. Cell Biol. 190, 511-521 (2010).

98. Baskaran, S. et al. Architecture and dynamics of the autophagic phosphatidylinositol 3-kinase complex. Elife 3, e05115 (2014).

99. Ma, M. et al. Cryo-EM structure and biochemical analysis reveal the basis of the functional difference between human PI3KC3-C1 and -C2. Cell Res. 27, 989-1001 (2017)

100. Su, H. et al. VPS34 acetylation controls its lipid kinase activity and the initiation of canonical and non-canonical autophagy. Mol. Cell 67, 907-921 (2017). e907.

101. Noda, N. N. et al. Structure of the novel C-terminal domain of vacuolar protein sorting 30/autophagy-related protein 6 and its specific role in autophagy. J. Biol. Chem. 287, 16256-16266 (2012).

102. Chang, $C$. et al. Bidirectional control of autophagy by BECN1 BARA domain dynamics. Mol. Cell 73, 339-353 (2019). e336.

103. Stjepanovic, G., Baskaran, S., Lin, M. G. \& Hurley, J. H. Vps34 kinase domain dynamics regulate the autophagic PI 3-kinase complex. Mol. Cell 67, 528-534 (2017). e523. 
104. Birgisdottir, A. B. et al. Members of the autophagy class III phosphatidylinositol 3-kinase complex I interact with GABARAP and GABARAPL1 via LIR motifs. Autophagy 15, 1333-1355 (2019).

105. Stromhaug, P. E., Reggiori, F., Guan, J., Wang, C. W. \& Klionsky, D. J. Atg21 is a phosphoinositide binding protein required for efficient lipidation and localization of Atg8 during uptake of aminopeptidase I by selective autophagy. Mol. Biol. Cell 15, 3553-3566 (2004).

106. Meiling-Wesse, K. et al. Atg21 is required for effective recruitment of Atg8 to the preautophagosomal structure during the Cvt pathway. J. Biol. Chem. 279, 37741-37750 (2004).

107. Obara, K., Sekito, T., Niimi, K. \& Ohsumi, Y. The Atg18-Atg2 complex is recruited to autophagic membranes via phosphatidylinositol 3-phosphate and exerts an essential function. J. Biol. Chem. 283, 23972-23980 (2008).

108. Zheng, J. X. et al. Architecture of the ATG2B-WDR45 complex and an aromatic Y/HF motif crucial for complex formation. Autophagy 13, 1870-1883 (2017).

109. Bakula, D. et al. WIPI3 and WIPI4 beta-propellers are scaffolds for LKB1-AMPKTSC signalling circuits in the control of autophagy. Nat. Commun. 8, 15637 (2017).

110. Chowdhury, S. et al. Insights into autophagosome biogenesis from structural and biochemical analyses of the ATG2A-WIPI4 complex. Proc. Natl Acad. Sci. USA 115, E9792-E9801 (2018).

111. Polson, H. E. et al. Mammalian Atg18 (WIPI2) localizes to omegasomeanchored phagophores and positively regulates LC3 lipidation. Autophagy 6 506-522 (2010).

112. Lu, Q. et al. The WD40 repeat Ptdlns(3)P-binding protein EPG-6 regulates progression of omegasomes to autophagosomes. Dev. Cell 21, 343-357 (2011).

113. Dove, S. K. et al. Svp1p defines a family of phosphatidylinositol 3,5-bisphosphate effectors. EMBO J. 23, 1922-1933 (2004).

114. Jeffries, T. R., Dove, S. K., Michell, R. H. \& Parker, P. J. Ptdlns-specific MPR pathway association of a novel WD40 repeat protein, WIPI49. Mol. Biol. Cell 15, 2652-2663 (2004)

115. Nair, U., Cao, Y., Xie, Z. \& Klionsky, D. J. Roles of the lipid-binding motifs of Atg18 and Atg21 in the cytoplasm to vacuole targeting pathway and autophagy. J. Biol. Chem. 285, 11476-11488 (2010).

116. Krick, R. et al. Structural and functional characterization of the two phosphoinositide binding sites of PROPPINs, a beta-propeller protein family. Proc. Natl Acad. Sci. USA 109, E2042-E2049 (2012).

117. Baskaran, S., Ragusa, M. J., Boura, E. \& Hurley, J. H. Two-site recognition of phosphatidylinositol 3-phosphate by PROPPINs in autophagy. Mol. Cell 47 339-348 (2012).

118. Watanabe, Y. et al. Structure-based analyses reveal distinct binding sites for Atg2 and phosphoinositides in Atg18. J. Biol. Chem. 287, 31681-31690 (2012).

119. Rieter, E. et al. Atg18 function in autophagy is regulated by specific sites within its beta-propeller. J. Cell Sci. 126, 593-604 (2013).

120. Busse, R. A. et al. Characterization of PROPPIN-phosphoinositide binding and role of loop 6CD in PROPPIN-membrane binding. Biophys. J. 108, 2223-2234 (2015).

121. Tamura, N. et al. Atg18 phosphoregulation controls organellar dynamics by modulating its phosphoinositide-binding activity. J. Cell Biol. 202, 685-698 (2013).

122. Gopaldass, N., Fauvet, B., Lashuel, H., Roux, A. \& Mayer, A. Membrane scission driven by the PROPPIN Atg18. EMBO J. 36, 3274-3291 (2017).

123. Maeda, S., Otomo, C. \& Otomo, T. The autophagic membrane tether ATG2A transfers lipids between membranes. Elife 8, e45777 (2019).

124. Scacioc, A. et al. Structure based biophysical characterization of the PROPPIN Atg18 shows Atg18 oligomerization upon membrane binding. Sci. Rep. 7, 14008 (2017).

125. Tamura, N. et al. Differential requirement for ATG2A domains for localization to autophagic membranes and lipid droplets. FEBS Lett. 591, 3819-3830 (2017).

126. Kotani, T., Kirisako, H., Koizumi, M., Ohsumi, Y. \& Nakatogawa, H. The Atg2Atg18 complex tethers pre-autophagosomal membranes to the endoplasmic reticulum for autophagosome formation. Proc. Natl Acad. Sci. USA 115, 10363-10368 (2018).

127. Valverde, D. P. et al. ATG2 transports lipids to promote autophagosome biogenesis. J. Cell Biol. 218, 1787-1798 (2019)

128. Tang, Z. et al. TOM40 targets Atg2 to mitochondria-associated ER membranes for phagophore expansion. Cell Rep. 28, 1744-1757 (2019). e1745.
129. Bozic, M. et al. A conserved ATG2-GABARAP family interaction is critical for phagophore formation. EMBO Rep. 21, e48412 (2020).

130. Gomez-Sanchez, R. et al. Atg9 establishes Atg2-dependent contact sites between the endoplasmic reticulum and phagophores. J. Cell Biol. 217, 2743-2763 (2018).

131. Osawa, T. et al. Atg2 mediates direct lipid transfer between membranes for autophagosome formation. Nat. Struct. Mol. Biol. 26, 281-288 (2019).

132. Kumar, N. et al. VPS13A and VPS13C are lipid transport proteins differentially localized at ER contact sites. J. Cell Biol. 217, 3625-3639 (2018).

133. Hanada, T. et al. The Atg12-Atg5 conjugate has a novel E3-like activity for protein lipidation in autophagy. J. Biol. Chem. 282, 37298-37302 (2007).

134. Otomo, C., Metlagel, Z., Takaesu, G. \& Otomo, T. Structure of the human ATG12 ATG5 conjugate required for LC3 lipidation in autophagy. Nat. Struct. Mol. Biol. 20, 59-66 (2013).

135. Fujioka, Y. et al. In vitro reconstitution of plant Atg8 and Atg12 conjugation systems essential for autophagy. J. Biol. Chem. 283, 1921-1928 (2008).

136. Sakoh-Nakatogawa, M. et al. Atg12-Atg5 conjugate enhances E2 activity of Atg3 by rearranging its catalytic site. Nat. Struct. Mol. Biol. 20, 433-439 (2013).

137. Romanov, J. et al. Mechanism and functions of membrane binding by the Atg5-Atg12/Atg16 complex during autophagosome formation. EMBO J. 31, 4304-4317 (2012).

138. Kaufmann, A., Beier, V., Franquelim, H. G. \& Wollert, T. Molecular mechanism of autophagic membrane-scaffold assembly and disassembly. Cell 156 469-481 (2014).

139. Fujita, N. et al. The Atg16 complex specifies the site of LC3 lipidation for membrane biogenesis in autophagy. Mol. Biol. Cell 19, 2092-2100 (2008).

140. Suzuki, K. et al. The pre-autophagosomal structure organized by concerted functions of APG genes is essential for autophagosome formation. EMBO J. 20, 5971-5981 (2001).

141. Mizushima, N. et al. Dissection of autophagosome formation using Apg5deficient mouse embryonic stem cells. J. Cell Biol. 152, 657-667 (2001).

142. Saitoh, T. et al. Loss of the autophagy protein Atg16L1 enhances endotoxininduced IL-1beta production. Nature 456, 264-268 (2008).

143. Itoh, T. et al. Golgi-resident small GTPase Rab33B interacts with Atg16L and modulates autophagosome formation. Mol. Biol. Cell 19, 2916-2925 (2008).

144. Gammoh, N., Florey, O., Overholtzer, M. \& Jiang, X. Interaction between FIP200 and ATG16L1 distinguishes ULK1 complex-dependent and -independent autophagy. Nat. Struct. Mol. Biol. 20, 144-149 (2013).

145. Nishimura, T. et al. FIP200 regulates targeting of Atg16L1 to the isolation membrane. EMBO Rep. 14, 284-291 (2013).

146. Fujita, N. et al. Recruitment of the autophagic machinery to endosomes during infection is mediated by ubiquitin. J. Cell Biol. 203, 115-128 (2013).

147. Juris, L. et al. PI3P binding by Atg21 organises Atg8 lipidation. EMBO J. 34, 955-973 (2015).

148. Harada, K. et al. Two distinct mechanisms target the autophagy-related E3 complex to the pre-autophagosomal structure. Elife 8, e43088 (2019).

149. Lystad, A. H. et al. Distinct functions of ATG16L1 isoforms in membrane binding and LC3B lipidation in autophagy-related processes. Nat. Cell Biol. 21, 372-383 (2019).

150. Dudley, L. J. et al. Intrinsic lipid binding activity of ATG16L1 supports efficient membrane anchoring and autophagy. EMBO J. 38, e100554 (2019).

151. Fletcher, K. et al. The WD40 domain of ATG16L1 is required for its noncanonical role in lipidation of LC3 at single membranes. EMBO J. 37, e97840 (2018).

152. Ichimura, Y. et al. A ubiquitin-like system mediates protein lipidation. Nature 408, 488-492 (2000).

153. Ichimura, $Y$. et al. In vivo and in vitro reconstitution of Atg8 conjugation essential for autophagy. J. Biol. Chem. 279, 40584-40592 (2004).

154. Ngu, M., Hirata, E. \& Suzuki, K. Visualization of Atg3 during autophagosome formation in Saccharomyces cerevisiae. J. Biol. Chem. 290, 8146-8153 (2015).

155. Sakoh-Nakatogawa, M., Kirisako, H., Nakatogawa, H. \& Ohsumi, Y. Localization of Atg3 to autophagy-related membranes and its enhancement by the Atg8-family interacting motif to promote expansion of the membranes. FEBS Lett. 589, 744-749 (2015).

156. Nath, S. et al. Lipidation of the LC3/GABARAP family of autophagy proteins relies on a membrane-curvature-sensing domain in Atg3. Nat. Cell Biol. 16, 415-424 (2014).

157. Hanada, T., Satomi, Y., Takao, T. \& Ohsumi, Y. The amino-terminal region of Atg3 is essential for association with phosphatidylethanolamine in Atg8 lipidation. FEBS Lett. 583, 1078-1083 (2009). 
158. Hervas, J. H. et al. Human ATG3 binding to lipid bilayers: role of lipid geometry, and electric charge. Sci. Rep. 7, 15614 (2017).

159. Li, Y. T. et al. A semisynthetic Atg3 reveals that acetylation promotes Atg3 membrane binding and Atg8 lipidation. Nat. Commun. 8, 14846 (2017).

160. Yi, C. et al. Function and molecular mechanism of acetylation in autophagy regulation. Science 336, 474-477 (2012).

161. Axe, E. L. et al. Autophagosome formation from membrane compartments enriched in phosphatidylinositol 3-phosphate and dynamically connected to the endoplasmic reticulum. J. Cell Biol. 182, 685-701 (2008).

162. Hayashi-Nishino, M. et al. A subdomain of the endoplasmic reticulum forms a cradle for autophagosome formation. Nat. Cell Biol. 11, 1433-1437 (2009).

163. Yla-Anttila, P., Vihinen, H., Jokita, E. \& Eskelinen, E. L. 3D tomography reveals connections between the phagophore and endoplasmic reticulum. Autophagy 5, 1180-1185 (2009).

164. Hailey, D. W. et al. Mitochondria supply membranes for autophagosome biogenesis during starvation. Cell 141, 656-667 (2010).

165. Ravikumar, B., Moreau, K., Jahreiss, L., Puri, C. \& Rubinsztein, D. C. Plasma membrane contributes to the formation of pre-autophagosomal structures. Nat. Cell Biol. 12, 747-757 (2010).

166. Ge, L., Melville, D., Zhang, M. \& Schekman, R. The ER-Golgi intermediate compartment is a key membrane source for the LC3 lipidation step of autophagosome biogenesis. Elife 2, e00947 (2013).

167. Puri, C., Renna, M., Bento, C. F., Moreau, K. \& Rubinsztein, D. C. Diverse autophagosome membrane sources coalesce in recycling endosomes. Cell 154, 1285-1299 (2013).

168. Puri, C. et al. The RAB11A-positive compartment is a primary platform for autophagosome assembly mediated by WIPI2 recognition of PI3P-RAB11A. Dev. Cell 45, 114-131 (2018). e118.

169. Dupont, N. et al. Neutral lipid stores and lipase PNPLA5 contribute to autophagosome biogenesis. Curr. Biol. 24, 609-620 (2014).

170. Uemura, T. et al. A cluster of thin tubular structures mediates transformation of the endoplasmic reticulum to autophagic isolation membrane. Mol. Cell Biol. 34, 1695-1706 (2014).

171. Duke, E. M. et al. Imaging endosomes and autophagosomes in whole mammalian cells using correlative cryo-fluorescence and cryo-soft X-ray microscopy (cryo-CLXM). Ultramicroscopy 143, 77-87 (2014).

172. Hamasaki, M. et al. Autophagosomes form at ER-mitochondria contact sites. Nature 495, 389-393 (2013)

173. Shpilka, T. et al. Lipid droplets and their component triglycerides and steryl esters regulate autophagosome biogenesis. EMBO J. 34, 2117-2131 (2015).

174. Nascimbeni, A. C. et al. ER-plasma membrane contact sites contribute to autophagosome biogenesis by regulation of local PI3P synthesis. EMBO J. $\mathbf{3 6}$, 2018-2033 (2017).

175. Stadel, D. et al. TECPR2 cooperates with LC3C to regulate COPII-dependent ER export. Mol. Cell 60, 89-104 (2015).

176. Tan, D. et al. The EM structure of the TRAPPIII complex leads to the identification of a requirement for COPII vesicles on the macroautophagy pathway. Proc. Natl Acad. Sci. USA 110, 19432-19437 (2013).

177. Davis, S. et al. Sec24 phosphorylation regulates autophagosome abundance during nutrient deprivation. Elife 5, e21167 (2016).

178. Shima, T., Kirisako, H. \& Nakatogawa, H. COPII vesicles contribute to autophagosomal membranes. J. Cell Biol. 218, 1503-1510 (2019).

179. Zhao, Y. G. et al. The ER-Localized transmembrane protein EPG-3NMP1 regulates SERCA activity to control ER-isolation membrane contacts for autophagosome formation. Mol. Cell 67, 974-989 (2017). e976.

180. Morita, K. et al. Genome-wide CRISPR screen identifies TMEM41B as a gene required for autophagosome formation. J. Cell Biol. 217, 3817-3828 (2018).

181. Tian, Y. et al. C. elegans screen identifies autophagy genes specific to multicellular organisms. Cell 141, 1042-1055 (2010).

182. Calvo-Garrido, J., King, J. S., Munoz-Braceras, S. \& Escalante, R. Vmp1 regulates Ptdlns3P signaling during autophagosome formation in Dictyostelium discoideum. Traffic 15, 1235-1246 (2014).

183. Bard, F. et al. Functional genomics reveals genes involved in protein secretion and Golgi organization. Nature 439, 604-607 (2006).

184. Calvo-Garrido, J., Carilla-Latorre, S., Lazaro-Dieguez, F., Egea, G. \& Escalante, R. Vacuole membrane protein 1 is an endoplasmic reticulum protein required for organelle biogenesis, protein secretion, and development. Mol. Biol. Cell 19, 3442-3453 (2008).

185. Morishita, H. et al. A critical role of VMP1 in lipoprotein secretion. Elife $\mathbf{8}$, e48834 (2019).
186. Tabara, L. C. et al. Vacuole membrane protein 1 marks endoplasmic reticulum subdomains enriched in phospholipid synthesizing enzymes and is required for phosphoinositide distribution. Traffic 19, 624-638 (2018).

187. Tabara, L. C. \& Escalante, R. VMP1 establishes ER-microdomains that regulate membrane contact sites and autophagy. PLOS ONE 11, e0166499 (2016).

188. Moretti, F. et al. TMEM41B is a novel regulator of autophagy and lipid mobilization. EMBO Rep. 19, e45889 (2018).

189. Shoemaker, C. J. et al. CRISPR screening using an expanded toolkit of autophagy reporters identifies TMEM41B as a novel autophagy factor. PLoS Biol. 17, e2007044 (2019).

190. Young, M. M. \& Wang, H. G. Sphingolipids as regulators of autophagy and endocytic trafficking. Adv. Cancer Res 140, 27-60 (2018).

191. Harvald, E. B., Olsen, A. S. \& Faergeman, N. J. Autophagy in the light of sphingolipid metabolism. Apoptosis 20, 658-670 (2015).

192. Li, Y. et al. The pleiotropic roles of sphingolipid signaling in autophagy. Cell Death Dis. 5, e1245 (2014).

193. Daido, S. et al. Pivotal role of the cell death factor BNIP3 in ceramide-induced autophagic cell death in malignant glioma cells. Cancer Res. 64, 4286-4293 (2004).

194. Scarlatti, F. et al. Ceramide-mediated macroautophagy involves inhibition of protein kinase B and up-regulation of beclin 1. J. Biol. Chem. 279 18384-18391 (2004).

195. Zeng, X., Overmeyer, J. H. \& Maltese, W. A. Functional specificity of the mammalian Beclin-Vps34 PI 3-kinase complex in macroautophagy versus endocytosis and lysosomal enzyme trafficking. J. Cell Sci. 119 , 259-270 (2006).

196. Guenther, G. G. et al. Ceramide starves cells to death by downregulating nutrient transporter proteins. Proc. Natl Acad. Sci. USA 105, 17402-17407 (2008).

197. Pattingre, S. et al. Role of JNK1-dependent BCl-2 phosphorylation in ceramide-induced macroautophagy. J. Biol. Chem. 284, 2719-2728 (2009).

198. Sims, K. et al. Kdo2-lipid A, a TLR4-specific agonist, induces de novo sphingolipid biosynthesis in RAW264.7 macrophages, which is essential for induction of autophagy. J. Biol. Chem. 285, 38568-38579 (2010).

199. Alexaki, A. et al. Autophagy regulates sphingolipid levels in the liver. J. Lipid Res. 55, 2521-2531 (2014).

200. Yamagata, M., Obara, K. \& Kihara, A. Sphingolipid synthesis is involved in autophagy in Saccharomyces cerevisiae. Biochem. Biophys. Res. Commun. 410, 786-791 (2011).

201. Lavieu, G. et al. Regulation of autophagy by sphingosine kinase 1 and its role in cell survival during nutrient starvation. J. Biol. Chem. 281, 8518-8527 (2006).

202. Moruno Manchon, J. F. et al. Cytoplasmic sphingosine-1-phosphate pathway modulates neuronal autophagy. Sci. Rep. 5, 15213 (2015).

203. Lepine, S. et al. Sphingosine-1-phosphate phosphohydrolase-1 regulates ER stress-induced autophagy. Cell Death Differ. 18, 350-361 (2011).

204. Chang, C. L. et al. S1P(5) is required for sphingosine 1-phosphate-induced autophagy in human prostate cancer PC-3 cells. Am. J. Physiol. Cell Physiol. 297, C451-C458 (2009).

205. Taniguchi, M. et al. Regulation of autophagy and its associated cell death by "sphingolipid rheostat": reciprocal role of ceramide and sphingosine 1phosphate in the mammalian target of rapamycin pathway. J. Biol. Chem. 287, 39898-39910 (2012).

206. Mitroi, D. N. et al. SGPL1 (sphingosine phosphate lyase 1) modulates neuronal autophagy via phosphatidylethanolamine production. Autophagy 13, 885-899 (2017).

207. Corcelle-Termeau, E. et al. Excess sphingomyelin disturbs ATG9A trafficking and autophagosome closure. Autophagy 12, 833-849 (2016).

208. Mishra, S. K. et al. CPTP: a sphingolipid transfer protein that regulates autophagy and inflammasome activation. Autophagy 14, 862-879 (2018).

209. Back, M. J. et al. Activation of neutral sphingomyelinase 2 by starvation induces cell-protective autophagy via an increase in Golgi-localized ceramide. Cell Death Dis. 9, 670 (2018).

210. Tan, S. H. et al. Induction of autophagy by palmitic acid via protein kinase Cmediated signaling pathway independent of MTOR (mammalian target of rapamycin). J. Biol. Chem. 287, 14364-14376 (2012).

211. Khan, M. J. et al. Inhibition of autophagy rescues palmitic acid-induced necroptosis of endothelial cells. J. Biol. Chem. 287, 21110-21120 (2012).

212. Martino, L. et al. Palmitate activates autophagy in INS-1E beta-cells and in isolated rat and human pancreatic islets. PLOS ONE 7, e36188 (2012). 
213. Las, G., Serada, S. B., Wikstrom, J. D., Twig, G. \& Shirihai, O. S. Fatty acids suppress autophagic turnover in beta-cells. J. Biol. Chem. 286 42534-42544 (2011).

214. Mei, S. et al. Differential roles of unsaturated and saturated fatty acids on autophagy and apoptosis in hepatocytes. J. Pharmacol. Exp. Ther. 339, 487-498 (2011).

215. O'Rourke, E. J., Soukas, A. A., Carr, C. E. \& Ruvkun, G. C. elegans major fats are stored in vesicles distinct from lysosome-related organelles. Cell Metab. 10, 430-435 (2009).

216. Niso-Santano, M. et al. Unsaturated fatty acids induce non-canonical autophagy. EMBO J. 34, 1025-1041 (2015).

217. Kohler, K. et al. A combined proteomic and genetic analysis identifies a role for the lipid desaturase Desat1 in starvation-induced autophagy in Drosophila. Autophagy 5, 980-990 (2009).

218. Ogasawara, Y. et al. Stearoyl-CoA desaturase 1 activity is required for autophagosome formation. J. Biol. Chem. 289, 23938-23950 (2014).

219. Ogasawara, Y., Kira, S., Mukai, Y., Noda, T. \& Yamamoto, A. Ole1, fatty acid desaturase, is required for Atg9 delivery and isolation membrane expansion during autophagy in Saccharomyces cerevisiae. Biol. Open 6, 35-40 (2017).

220. Sauvat, A. et al. Trans-fats inhibit autophagy induced by saturated fatty acids. EBioMedicine 30, 261-272 (2018)

221. Dall'Armi, C. et al. The phospholipase D1 pathway modulates macroautophagy. Nat. Commun. 1, 142 (2010).

222. Bae, E. J. et al. Phospholipase D1 regulates autophagic flux and clearance of alpha-synuclein aggregates. Cell Death Differ. 21, 1132-1141 (2014).

223. Cai, M. et al. Phospholipase D1-regulated autophagy supplies free fatty acids to counter nutrient stress in cancer cells. Cell Death Dis. 7, e2448 (2016).

224. Holland, P. et al. HS1BP3 negatively regulates autophagy by modulation of phosphatidic acid levels. Nat. Commun. 7, 13889 (2016).
225. Jang, Y. H., Choi, K. Y. \& Min, D. S. Phospholipase D-mediated autophagic regulation is a potential target for cancer therapy. Cell Death Differ. 21, 533-546 (2014).

226. Li, D. et al. Storage lipid synthesis is necessary for autophagy induced by nitrogen starvation. FEBS Lett. 589, 269-276 (2015).

227. Velazquez, A. P., Tatsuta, T., Ghillebert, R., Drescher, I. \& Graef, M. Lipid dropletmediated ER homeostasis regulates autophagy and cell survival during starvation. J. Cell Biol. 212, 621-631 (2016).

228. Shatz, O., Holland, P., Elazar, Z. \& Simonsen, A. Complex relations between phospholipids, autophagy, and neutral lipids. Trends Biochem. Sci. 41, 907-923 (2016).

229. Cheng, J. et al. Yeast and mammalian autophagosomes exhibit distinct phosphatidylinositol 3-phosphate asymmetries. Nat. Commun. $\mathbf{5}$ 3207 (2014).

230. Baba, T., Toth, D. J., Sengupta, N., Kim, Y. J. \& Balla, T. Phosphatidylinositol 4,5-bisphosphate controls Rab7 and PLEKHM1 membrane cycling during autophagosome-lysosome fusion. EMBO J. 38, e100312 (2019).

231. Turco, E. \& Martens, S. Insights into autophagosome biogenesis from in vitro reconstitutions. J. Struct. Biol. 196, 29-36 (2016)

232. Bahrami, A. H., Lin, M. G., Ren, X., Hurley, J. H. \& Hummer, G. Scaffolding the cup-shaped double membrane in autophagy. PLoS Comput. Biol. 13, e1005817 (2017).

233. Knorr, R. L., Dimova, R. \& Lipowsky, R. Curvature of double-membrane organelles generated by changes in membrane size and composition. PLoS ONE 7, e32753 (2012).

234. Yamamoto, $H$. et al. The Intrinsically disordered protein Atg13 mediates supramolecular assembly of autophagy initiation complexes. Dev. Cell $\mathbf{3 8}$, 86-99 (2016). 